\title{
Unsteady Fluid Physics and Surrogate Modeling of Low Reynolds Number, Flapping Airfoils
}

\author{
Patrick C. Trizila ${ }^{1}$, Chang-kwon Kang ${ }^{2}$, Miguel R. Visbal ${ }^{3}$, and Wei Shyy ${ }^{4}$ \\ ${ }^{1,2,4}$ Department of Aerospace Engineering, University of Michigan, Ann Arbor, Michigan 48109 \\ ${ }^{3}$ U.S. Air Force Research Laboratory, Wright-Patterson Air Force Base, Ohio 45433
}

\begin{abstract}
To improve our understanding of the fluid physics related to micro air vehicles (MAVs), the current work investigates the low chord Reynolds $(\mathrm{Re})$ number, between $10^{2}$ and $10^{3}$, fluid physics of a 2D flapping airfoil via direct numerical simulation and surrogate modeling. Addressed are the impacts of kinematic parameters and Re number under freestream/hovering conditions. The kinematic parameters include plunging amplitude, angular amplitude, and pitching/plunging phase angle. Composite surrogate models are constructed and global sensitivity evaluations of these variables are analyzed. Wake capture, delayed stall, and interaction with a jet-like flow feature all influence the performance of the airfoil. It is found that the plunging amplitude and reduced frequency play a surprisingly small role in determining the airfoil performance in the design space examined. Interestingly, in normal hovering studied here, the kinematic variables are largely uncoupled, and while the aerodynamics are complex, that the cumulative effect can be largely explained with a linear superposition of individual influences. Furthermore, delayed stall and the jet interaction exhibit major influence on the overall lift. As expected, the kinematics requiring the least amount of power occurred at high angular amplitudes, with minimum delayed stall and angle of attack at the maximum translational velocity $(\Phi=90)$.
\end{abstract}

\section{Nomenclature}

$$
\mathrm{C}_{\mathrm{D}} \quad=\quad \text { drag coefficient }
$$

$\mathrm{C}_{\mathrm{L}}=$ lift coefficient

$\mathrm{C}_{\mathrm{L} \text {, vis }}=\quad$ viscous component of the lift coefficient

$\mathrm{c}=$ chord length

$\mathrm{D}=$ partial variance

$\mathrm{dt}=$ timestep

$\mathrm{f}=$ frequency of oscillation

$\mathrm{h}=$ translational position

$\mathrm{h}_{\mathrm{a}}=$ plunging amplitude

$\mathrm{k}=$ reduced frequency

$\mathrm{L}_{\text {ref }}=$ reference length

MAV $=$ micro air vehicle

$\mathrm{N}=$ number of design variables

$\mathrm{P}_{\text {req }}=$ power required

\footnotetext{
${ }^{1}$ Graduate Student, Department of Aerospace Engineering, University of Michigan, Member AIAA.

${ }^{2}$ Graduate Student, Department of Aerospace Engineering, University of Michigan, Member AIAA.

${ }^{3}$ Technical Area Leader, Computational Sciences Branch, AFRL/RBAC, Associate Fellow AIAA.

${ }^{4}$ Clarence L. "Kelly" Johnson Collegiate Professor and Chair, Department of Aerospace Engineering, University of Michigan, Fellow AIAA.
} 


$\begin{array}{lll}\mathrm{p} & = & \text { pressure } \\ \mathrm{PRESS}= & \text { prediction sum of squares; a measure of the cross validation errors } \\ \mathrm{Re} & = & \text { Reynolds number } \\ \mathrm{T} & = & \text { period of oscillation } \\ \mathrm{t} & = & \text { time } \\ \mathrm{U}_{\max } & = & \text { maximum velocity } \\ \mathrm{U}_{\mathrm{ref}} & = & \text { reference velocity } \\ \mathrm{u} & = & \text { velocity } \\ \alpha & = & \text { angular position } \\ \alpha_{0} & = & \text { initial angular position } \\ \alpha_{\mathrm{a}} & = & \text { angular amplitude } \\ \mathrm{v} & = & \text { kinematic viscosity } \\ \rho & = & \text { density } \\ \Phi & = & \text { phase lag } \\ <\mathrm{x}> & = & \text { time averaged value of } \mathrm{x}\end{array}$

\section{Introduction}

$\mathrm{T}$ he term micro air vehicle generally refers to a vehicle whose maximum dimension is less than $15 \mathrm{~cm}^{1}$. The three broad classifications used to distinguish them are: (i) the fixed wing variety ${ }^{1}$, which has been successfully designed and flown with a maximum dimension of smaller than $10 \mathrm{~cm}$ and operates at the chord Reynolds number of $10^{4}-10^{5}$; (ii) the rotor based models which roughly resemble helicopters ${ }^{2}$; (iii) the flapping wing family, inspired by behaviors seen in nature ${ }^{3}$. While the flapping wing concept can be adopted for larger flyers; a main interest in MAVs is for smaller flyers of several $\mathrm{cm}$ or less and the chord Reynolds number between $10^{2}-10^{4}$. Various aerodynamics and fluid-structure interaction aspects relevant to MAVs can be found in a number of publications, e.g., Refs. 3-11. To achieve the performance goals necessary to realize flapping wing vehicles of robust performance, further progress in understanding and manipulation of the flow physics will be needed as will guiding principles to help in design of proper wing kinematics.

While the ultimate goal of this line of research seeks the 3D kinematics yielding the best performance for flapping wings with varying sizes, frequency, structural complexity, and capable of handling forward flight, hovering and wind gust scenarios, the immediate task presented in this study is to better understand the interaction and effects of the unsteady flow mechanisms and compare potential kinematic combinations with the use of surrogate models. There has much progress made related to these interests. Reynolds number effects have been examined previously, e.g., Ref 12, and one such consequence, the asymmetric forward and back stroke, is seen in the current context as a consequence of interactions with the jet like flow feature present. The sinusoidal hovering kinematics utilized here have been used in former studies as well (see Refs 13 and 14), and yet there are still questions even in the simplified 2D domain. What constitutes "good" kinematics? This is a context specific question, e.g., the measure of merit may be lift, but then the next relevant question is why, and at what cost (power consumption). Why are certain combinations of variables better than others and can any general trends be stated? This is merely a starting point. After a stronger foundation has been set other aspects can be addressed. Transitional effects ${ }^{13}$ are relevant at Reynolds numbers seen by MAVs and are one of the open challenges in the field. The 3D effects at relatively low aspect ratios are quite important. The traditional wing tip vortices ${ }^{14}$ are present but there are also highly complex flow field interactions which can be seen in Refs 15 and 16 for computations of a flapping hawkmoth. All of these are possible avenues that can be eventually integrated into the current framework. Here we build upon the aspects pertaining to $2 \mathrm{D}$ hovering. Relevant studies here have been steadily increasing for quite some time, early numerical computations can be found in Ref 17 and experimental work later performed in Ref 18 .

Surrogate modeling is one of the optimization methodologies used in engineering environments. Ref 19 presents an overview and highlights the strengths and issues in using surrogate based analysis while Ref. 20 specifically addresses shortcomings of the experimental designs. Surrogate modeling provides an efficient method for mining information from limited data sets which is usually expensive, be it in computational or experimental costs. Examples of engineering applications include shape optimization using response surfaces ${ }^{21}$ as well as other surrogate models $^{22}$ in the design of rocket injectors and supersonic turbines ${ }^{23}$. It is seen that alternatives to gradient based optimizations are needed, and these examples provide empirical evidence of the utility in using surrogate models. In the present study, they are used to get an idea of how the design space behaves away from the known 
data points. Not only does this help clarify the general trends, but when the design of experiment (DOE) is done properly, the result is more efficient use of computational resources.

This study starts with a verification exercise and then moves to the kinematic consequences on the unsteady flight mechanisms. Namely, delayed stall, largely resulting from the leading edge vortex (LEV), wake capturing techniques, and a persistent interaction with a jet-like flow feature will interact and greatly influence the wing's aerodynamic performance. The scope of the present investigation is restricted, and various simplifications were made in the analysis in order to develop a methodology for quantitatively assessing a design variable's impact. The airfoil is taken to be an ellipse with $15 \%$ thickness, while the kinematics are $2 \mathrm{D}$ and restricted to limit the degrees of freedom. The normal hovering motion used is confined to sinusoidal plunging in one dimension and sinusoidal pitching motions. A main effort is to build a framework for classifying the fully 3D unsteady aerodynamics; these simplifications provide a logical stepping stone in that direction. This leaves three degrees of freedom which are systematically probed with the aid of computational fluid dynamics (CFD) simulations along with the surrogate modeling and sensitivity analysis tools. The time averaged lift coefficient, $\left\langle\mathrm{C}_{\mathrm{L}}\right\rangle$, and power required are used as the objective functions for the normal hovering cases without freestream whereas $\left\langle\mathrm{C}_{\mathrm{L}}\right\rangle$ and its viscous component $<\mathrm{C}_{\mathrm{L}, \mathrm{vis}}>$ are used in the freestream cases. Note that while the surrogate modeling technique is borrowed directly from the optimization community, the current purpose is to probe the flow physics and not necessarily find an optimal design.

\section{Approaches and Tools}

\section{A. Computational Models}

The governing equations are the laminar, unsteady, Navier-Stokes equations with constant transport properties; the incompressible versions are shown in Eqs. (1) and (2) written in indicial form.

$$
\begin{gathered}
\frac{\partial}{\partial x_{j}}\left(u_{j}\right)=0 \\
\frac{\partial}{\partial t}\left(u_{i}\right)+\frac{\partial}{\partial x_{j}}\left(u_{j} u_{i}\right)=-\frac{1}{\rho} \frac{\partial p}{\partial x_{i}}+v \frac{\partial}{\partial \mathrm{x}_{j}}\left(\frac{\partial u_{i}}{\partial x_{j}}\right)
\end{gathered}
$$

Here $u_{i}$ is the velocity component in the $\mathrm{i}^{\text {th }}$ direction, $x_{i}$ is the $\mathrm{i}^{\text {th }}$ Cartesian position vector, $t$ is time, $\rho$ is density, $p$ is pressure, and $v$ is the kinematic viscosity. A rule-based software ${ }^{24}$, Loci-STREAM ${ }^{25}$ is used to calculate the solutions. Loci-STREAM is a three-dimensional, unstructured, pressure based solver. The present calculations use implicit first or second order time stepping (the first order technique is adopted in this study). The convection terms are treated using the second order upwind scheme ${ }^{26,27}$ while pressure and viscous terms are treated using second order central differencing schemes. The system of equations resulting from the linearized momentum equations are fast to converge ${ }^{27}$ and are handled with the symmetric Gauss-Seide ${ }^{28}$ solver which has relatively low memory requirements. The pressure equation, derived in Refs. 25 and 29, is slower to converge, and is handled by the PETSc Krylov $^{28}$ solvers with Jacobi preconditioning. The Loci framework is by design highly parallelizable and can take advantage of many processors.

The translational and rotational airfoil/grid motions are dictated by Eqs. (3) and (4).

$$
\begin{gathered}
h(t)=h_{a} \sin (2 \pi f t) \\
\alpha(t)=\alpha_{0}-\alpha_{a} \sin (2 \pi f t+\phi)
\end{gathered}
$$

Here $h(t)$ and $h_{a}$ are the translational position and plunging amplitude respectively. The angular orientation, initial angle, and angular amplitude are $\alpha(t), \alpha_{0}$, and $\alpha_{a}$ respectively. The pitching is about the center of the rigid airfoil; this is an ellipse having a $15 \%$ thickness for all cases under consideration. The phase lag between the two motions is $\Phi$, and the frequency is denoted $f$ whereas the time is again $t$. While there are a few choices in how to accommodate these kinematics computationally, the current implementation forces the grid to rotate and translate with airfoil. The geometric conservation law ${ }^{30}(\mathrm{GCL})$, a necessary consideration in domains with moving boundaries, is satisfied ${ }^{31}$. The boundary condition applied to all outer boundaries is the incompressible inlet with density and velocity specified. 


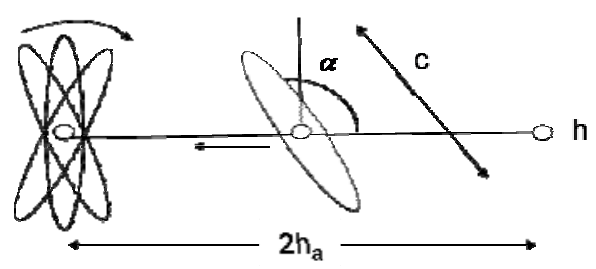

Figure 1. Illustration of the kinematic parameters for normal hovering.

Due to the kinematic constraints there are only two relevant non-dimensional groups in the incompressible case. The plunging amplitude to chord ratio, $2 h_{a} / c$, and the Reynolds number:

Rewritten for hovering the $R e$ becomes:

$$
R e=\frac{U_{r e f} L_{r e f}}{v}
$$

$$
R e_{\text {hovering }}=\frac{\left(2 \pi f h_{a}\right) c}{v}
$$

The reference velocity in this case is the maximum translational velocity, defined by the flapping frequency, $f$, and the plunging amplitude, $h_{a}$. Since $R e$ is being held constant, $h_{a}$ and $f$ are not independent.

In the case of freestream, the flapping $R e$ is matched, as well as the $R e$ based on the freestream velocity:

$$
R e_{\text {freestream }}=\frac{U_{\infty} C}{v}
$$

Note that the reduced frequency, $k$, is not emphasized here as, in the absence of a freestream, it contains the same information as the plunging amplitude ratio.

$$
k_{\text {hovering }}=\frac{2 \pi L_{r e f}}{2 U_{r e f}}=\frac{2 \pi f c}{2\left(2 \pi f h_{a}\right)}=\frac{c}{2 h_{a}}
$$

The three quantities that we can independently vary are $h_{a}, \alpha_{a}$, and $\Phi$.

\section{B. Surrogate Modeling}

The surrogate modeling techniques are used to gain a better understanding of the impact of the design variables. The various time histories give a telling story as to what is happening for each combination, but illustrating the overall impact is not straight forward, even when limited to three degrees of freedom. Surrogate modeling provides a way to stand back from the trees and see the forest if you will. The process is split into three main parts. The first is constructing the design of experiment (DOE) $)^{32}$, or method for choosing how many and for which points to run full CFD simulations. These then provide the necessary data for the fitting of the surrogate models which can be used to approximate a quantity of choice at arbitrary points within the design space. The third piece of the process is a sensitivity analysis ${ }^{19}$ which can be used to quantify the importance of each design variable, and in some cases eliminate them from consequent refinement iterations.

\section{Design Space}

The range of the variables was chosen after considering Refs. 33,34, and 35 which tabulated actual measurements from various species, some of which are reproduced previously in Table 1 . The bounds for the simulations are listed in Table 2, though it is worth noting that $\phi<90^{\circ}$, also referred to as delayed rotation, is not expected to be found in nature for hovering flight as the airfoil is flying upside for portions of the cycle. These cases were included to provide some symmetry for the other extreme, $\phi>90^{\circ}$ or advanced rotation. Furthermore, measurements regarding $\alpha_{a}$ are also difficult to come by, though comments found in Ref. 35 would imply that the choice of bounds, Table 2, for this variable are reasonable. 


\begin{tabular}{|l|c|c|c|c|}
\hline & $c(\mathrm{~cm})$ & $f(\mathrm{~Hz})$ & $2 h_{a} / \mathrm{l}$ & $R e_{\text {wing }}$ \\
\hline $\begin{array}{l}\text { Fruit Fly: } \\
\text { Drosophila virilis }\end{array}$ & 0.15 & 240 & 3.5 & 250 \\
\hline $\begin{array}{l}\text { Honey Bee: } \\
\text { Apis mellifica }\end{array}$ & 0.43 & 240 & 2.8 & 4900 \\
\hline $\begin{array}{l}\text { Bumble Bee: } \\
\text { Bombus terrestris }\end{array}$ & 0.73 & 156 & 2.8 & 6400 \\
\hline $\begin{array}{l}\text { Hummingbird: } \\
\text { Archilochus colubris }\end{array}$ & 1.5 & 52 & 3.6 & 6700 \\
\hline $\begin{array}{l}\text { Hawkmoth: } \\
\text { Manduca Sexta }\end{array}$ & 2.5 & 27.3 & 3.6 & 15000 \\
\hline $\begin{array}{l}\text { Hummingbird: } \\
\text { Patagona gigas }\end{array}$ & 4.3 & 15 & 2.6 & \\
\hline
\end{tabular}

Table 1. Selected data ${ }^{33,34}$ on the time and length scales encountered in nature. The examples listed do not provide upper or lower bounds for any of the categories listed, but do provide a window which captures many of the insects and animals capable of hovering flight.

\begin{tabular}{|l|c|c|}
\hline Parameter & Minimum & Maximum \\
\hline $2 h_{a} / c$ & 2.0 & 4.0 \\
\hline$\alpha_{a}$ & $45^{\circ}$ & $80^{\circ}$ \\
\hline$\Phi$ & $60^{\circ}$ & $120^{\circ}$ \\
\hline
\end{tabular}

Table 2. Minimum and maximum values of the plunging ratio, angular amplitude, and phase lag simulated in the surrogate modeling exercise.

\section{Design of Experiment}

The DOE used a face centered cubic design (FCCD ${ }^{23}$ and then Latin hypercube sampling (LHS) ${ }^{19}$ to appropriately fill in the remainder of the design space. A $2^{\text {nd }}$ order polynomial response surface construction has $(\mathrm{N}+1)(\mathrm{N}+2) / 2$ coefficients, $\mathrm{N}$ being the number of variables, and in general, one wants twice this many data points for a proper curve fit. A FCCD design provides $2^{\mathrm{N}}+2 \mathrm{~N}+1$ points: $2^{\mathrm{N}}$ corner points, $2 \mathrm{~N}$ face points, and one center point. Thus for three design variables, FCCD provides 15 of the 20 points required. The LHS then provides a method for efficiently choosing the rest of the points by maximizing the distance between the added points, though by no means is it the only alternative ${ }^{19}$. To balance the computational expense these simulations were carried out an $81 \times 81$ grid, a resolution which was not strictly grid independent, but sufficient to capture the relevant behavior immediately surrounding the airfoil as seen in Figure 2. Tabulation of the design points and objective function values can be found in the appendix, Table A1.

\section{Composite Surrogates}

Which surrogate model(s) to use is an interesting challenge in and of itself. The current approach utilizes the current state of the art work of Refs 36 and 37. Here a number of different surrogate models are constructed and are evaluated based on their respective cross-validation errors, namely PRESS ${ }^{37}$. In this fashion no extra test points are needed, rather the models are constructed with one less training point and the deviation of this point from the constructed surrogate is used calculate one component of the PRESS. Each of the training points is treated in a similar manor and one can subsequently quantify how well the respective surrogate model fits. As Ref 37 illustrates for problems in lower dimensions, using the best PRESS surrogate model might be justified, whereas in higher dimensions it is much riskier to do so.

The current setup evaluates Kriging ${ }^{19}, 2^{\text {nd }}$ order polynomial response (PRS) ${ }^{38}$, radial basis neural network $(\mathrm{RBNN})^{39}$, and 6 support vector regression (SVR) ${ }^{40}$ models noted in Table 3 . The two best models in the current context, as measured by those exhibiting the lowest PRESS values, are the SVR model using a full spline kernel and Kriging. 


\begin{tabular}{|l|l|l|}
\hline Model & Comment 1 & Comment 2 \\
\hline Kriging & Linear Regression Model & Gaussian Correlation Model \\
\hline PRS & $2^{\text {nd }}$ Order Polynomial & ----- \\
\hline RBNN & Max Neurons =1000 & ---- \\
\hline SVR1 & Linear Spline Kernel & $\begin{array}{l}\text { Full- infinity as upper bound } \\
\text { (non-separable case) }\end{array}$ \\
\hline SVR2 & Linear Spline Kernel & Short- finite upper bound \\
\hline SVR3 & Exponential Kernel & Full \\
\hline SVR4 & Exponential Kernel & Short \\
\hline SVR5 & Gaussian Kernel & Full \\
\hline SVR6 & Gaussian Kernel & Short \\
\hline
\end{tabular}

Table 3. Defining surrogate model traits.

\section{Global Sensitivity Analysis}

The global sensitivity analysis is in general useful for: (i) Determining if a variable is particularly influential in the design space, if not perhaps the variable can be fixed and the degrees of freedom and complexity of the problem reduced. (ii) Ranking the importance of the design variables. (iii) Quantifying the degree of coupling between design variables. For example, is the influence on the design space mostly an individual effort, or is there an effect caused by the interaction of variables?

Sobol's method ${ }^{19}$ is used to for the global sensitivity evaluations. The surrogate model can be written as:

$$
f(x)=f_{0}+\sum_{i} f_{i}\left(x_{i}\right)+\sum_{i<j} f_{i j}\left(x_{i}, x_{j}\right)+\cdots
$$

Once this decomposition has been calculated the total variance,

$$
D=\int f(\boldsymbol{x})^{2} d \boldsymbol{x}-f_{0}^{2}
$$

and partial variances, e.g.,

$$
D_{1}=\int f_{1}(\boldsymbol{x}) d x_{1} d x_{2} d x_{3}
$$

can be calculated. In this fashion, individual contributions, such as $\mathrm{D}_{1} / \mathrm{D}$, or combinations of variables, e.g., $\mathrm{D}_{12} / \mathrm{D}$, can be quantified, effectively capturing the sensitivity of the variable(s) under consideration.

\section{Results and Discussion}

We now explain the investigation of flapping wing kinematics and the individual and combined effects of the plunging amplitude, angular amplitude, and phase lag under hovering and freestream conditions at $\mathrm{Re}=100$ and $\mathrm{Re}$ $=1000$.

\section{A. Grid and Timestep Sensitivity}

Prior to running the DOE simulations, grid and timestep sensitivity tests were performed for a representitive case. As can be seen in Figure 2, grid convergence is attained at resolutions of 161x161 and higher. The results shown here are broadly consistent with those obtained using another computational algorithm, based on higher-order finite difference schemes ${ }^{41,42}$. For the timestep plots, a case was run at the finest timestep resolution and then restarted with the timesteps noted. Convergence at a timestep dictated that more subiterations were used for the coarser timesteps. Because of the computational cost involved in running all of the simulations as well as the expected level of accuracy output by the surrogate models, the error encountered with the $81 \mathrm{x} 81$ grids was deemed acceptable. This is put in the context of the PRESS calculations,see Appendix, and independent testing points which suggest that errors were less than $10 \%$. A timestep of T/1000 was chosen because it provided the best accuracy and simulation walltime combination (note: $\mathrm{dt}=\mathrm{T} / 500$ computations required more walltime to reach comparable solutions at $\mathrm{dt}=\mathrm{T} / 1000$ ). As can be verified in Figure 3, the force histories do not immediately assume repeatable amplitudes. The changes between cycles had largely plateaued by the $15^{\text {th }}$ cycle for the $81 \times 81$ grid resolution, and accordingly the time averaged values for cases without freestream were taken over cycles 15 through 20 .

For the freestream cases, a prominent flow direction made numerical convergence easier, and the same grid and timestep choices were used. For Reynolds number cases at 1000, the normal hovering cases, without freestream, did not provide a repeatable force history as will be shown later. The vortex dynamics quickly lead to more and more 
chaotic interactions, providing little meaning in the surrogate model exercise. With the presence of a freestream at $\operatorname{Re}=1000$, the force histories were again repeatable, though a finer timestep, $\mathrm{dt}=\mathrm{T} / 2000$, was implemented.

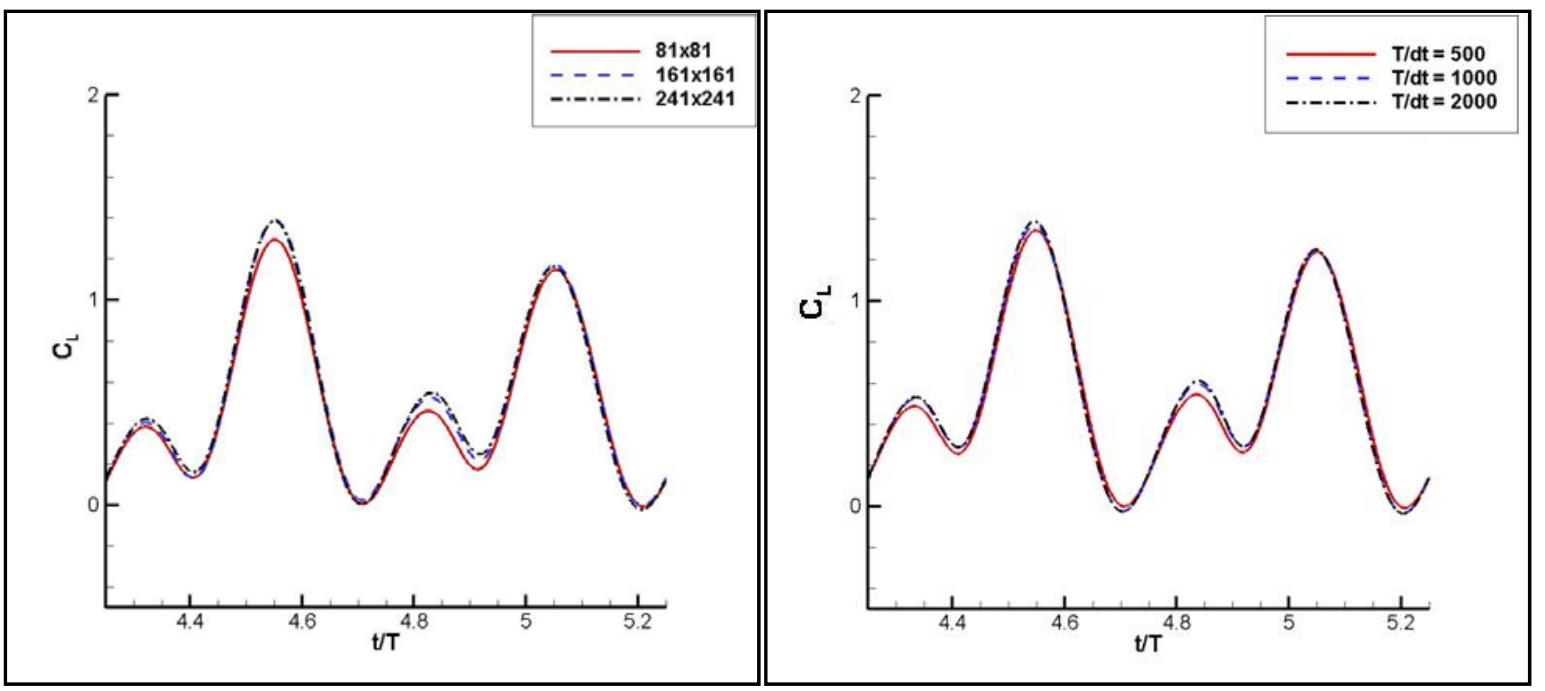

Figure 2. Left: The grid sensitivity for normal hovering at $\operatorname{Re}=75,2 \mathrm{ha} / \mathrm{c}=2.8, \alpha_{\mathrm{a}}=45, \varphi=90$. Right: Timestep sensitivity once proper convergence criteria were enforced for $\operatorname{Re}=100,2 \mathrm{ha} / \mathrm{c}=2.8, \alpha_{\mathrm{a}}=45, \varphi=$ 90.

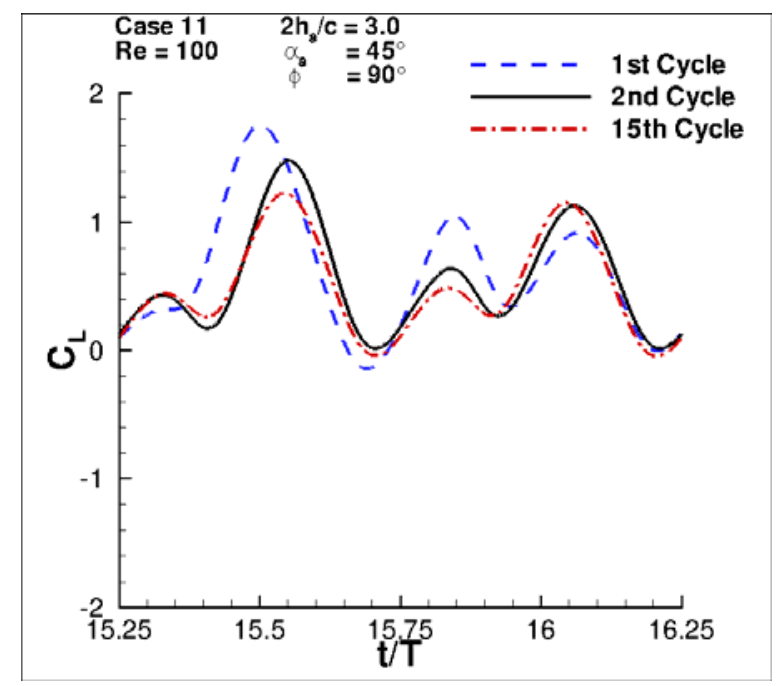

Figure 3. Illustration of the magnitude of the jet influence on airfoil performance. The jet's structure has not reached a stable configuration during the first few cycles and therefore, most of the discrepancy can be attributed to this unsteady flow feature.

\section{B. Force Interpretation}

To better understand the implications and limitations of the surrogate modeling results an example is presented of a representative normal hovering case at a Re of 100, see Figure 4. The objective functions for these cases will be the time integrated $C_{L}$ and an approximation to the non-dimensional time averaged power required which is calculated by multiplying the non-dimensional translational velocity by the $\mathrm{C}_{\mathrm{D}}$. 

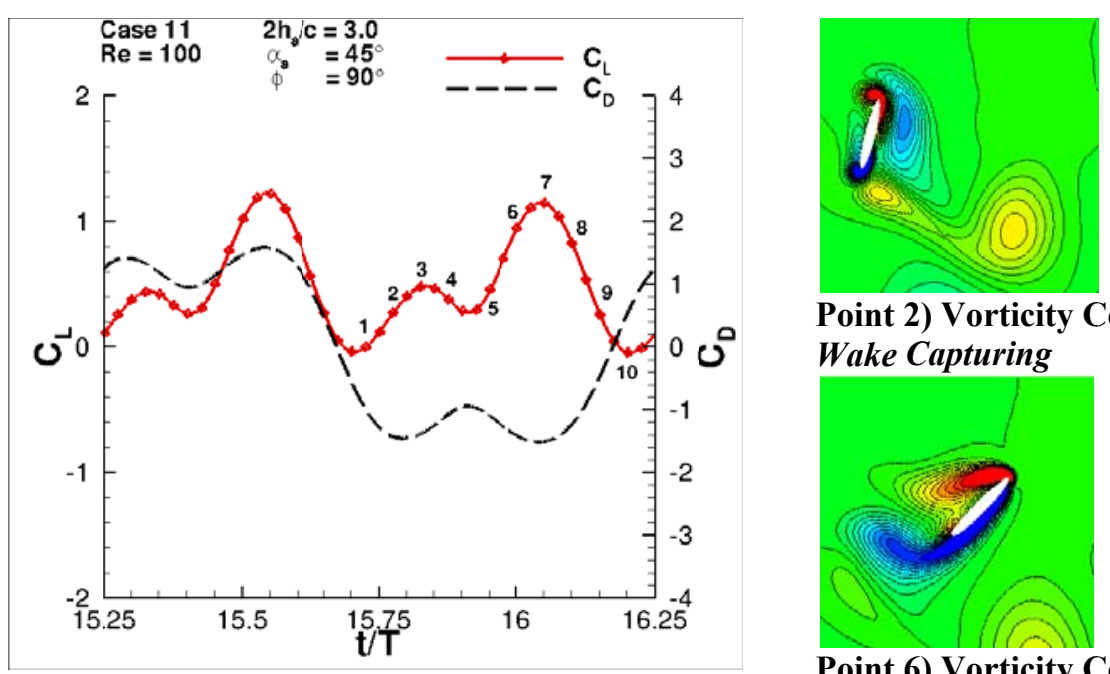

\section{Point 2) Vorticity Contours Wake Capturing}

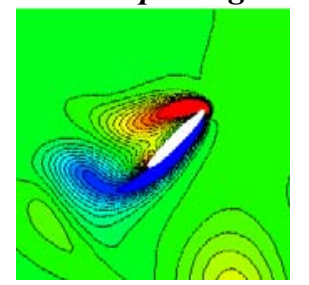

Point 6) Vorticity Contours

Delayed Stall
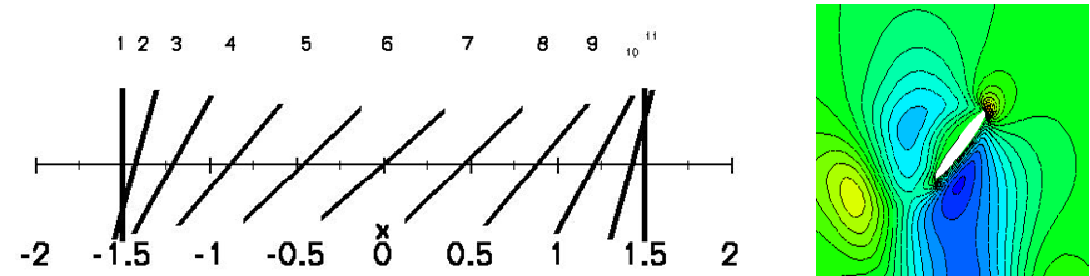

\section{Point 6) Vertical Velocity \\ Jet Interaction}

Figure 4. Illustration of the force histories over a normal hovering cycle for the parameters of $2 \mathrm{ha} / \mathrm{c}=3.0, \alpha_{\mathrm{a}}$ $=45$, and $\Phi=90$ and the corresponding airfoil positions. Three flowfield shots illustrating the unsteady aerodynamics are emphasized.

The discussion following is generally applicable to the cases where $\Phi=90$ with slight modifications. For cases where the phase lag dictates advanced rotation $(\Phi>90)$ or delayed rotation $(\Phi<90)$, the same ideas can be extended though like the parameter suggests, the translation and rotation will be out of phase. The cycle can be broken up into three overlapping regions defined by the unsteady flow mechanisms present.

The first region starts at point 1, once again referring to Figure 4, which is near a local minimum in the lift. As the airfoil is vertical at this point, one would generally expect zero lift. As time continues the airfoil turns back into its previous trajectory which is commonly referred to as wake capturing, points 1,2 , and 3 . The peak seen at point 3 will be referred to as the wake capturing peak. Flow field shots of vorticity, Figure 5 , demonstrate the nomenclature more clearly.

The second and third unsteady flow features overlap significantly. The most commonly known is the delayed stall phenomena here resulting from a leading edge vortex, Figure 6. Specifically, a vortex forms behind the leading edge of the airfoil causing a low pressure region and enhancing lift. Note that in the case illustrated, higher lift is achieved at angles of attack of 45 degrees, an angle well beyond the steady state stall. In cases with higher angular amplitudes, and therefore lower angles of attack, the peak at points 7 and 8 can be reduced significantly because the orientation of the airfoil is not able to promote LEV formation (see Figure 11). 

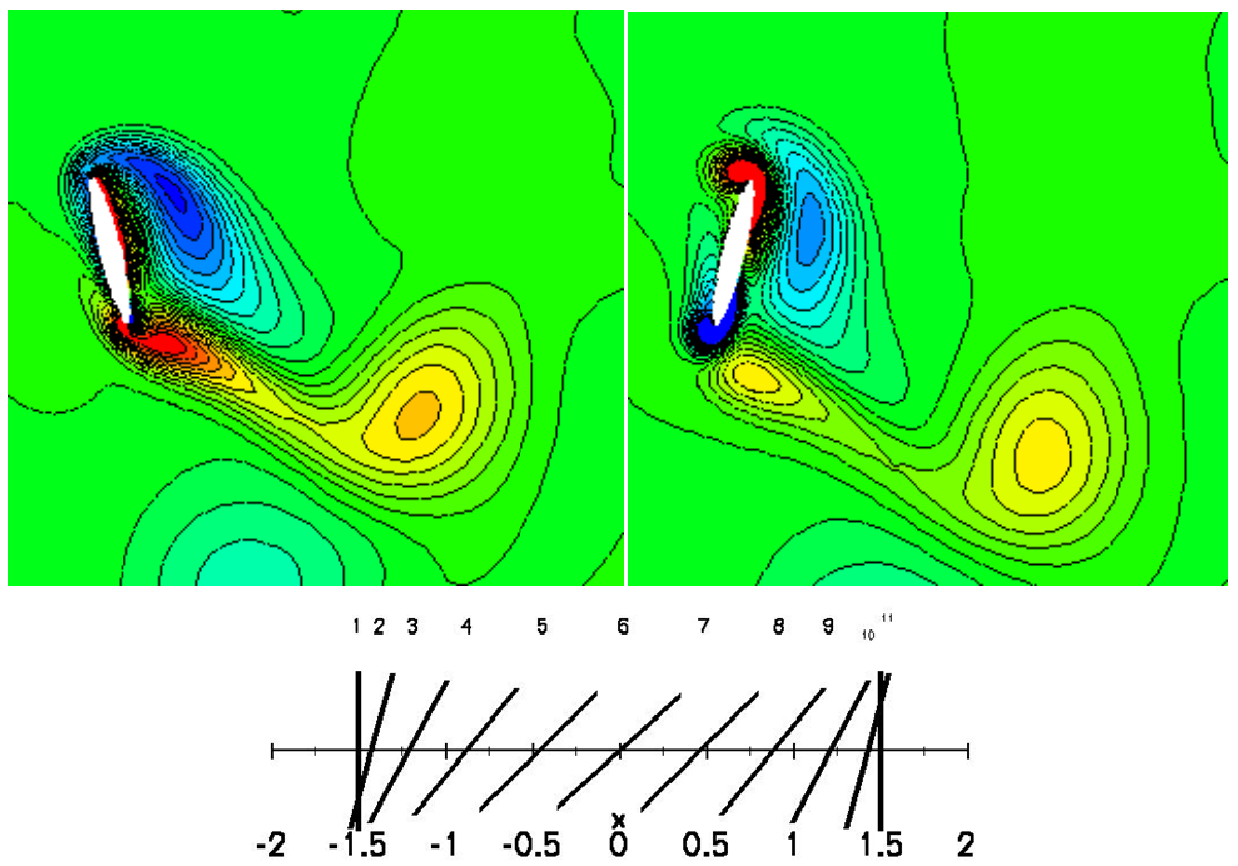

Figure 5. Wake Capturing: Illustration of vorticity countours (red: counter-clockwise, blue: clockwise) and the wake capturing portion of the cycle where the airfoil turns back onto its wake possibly taking advantage of the unsteady aerodynamics. These plots would correspond to points on either side of point 1 . The unmarked point 0 on the left which belongs to the end of the backstroke, and point 2 on the right.
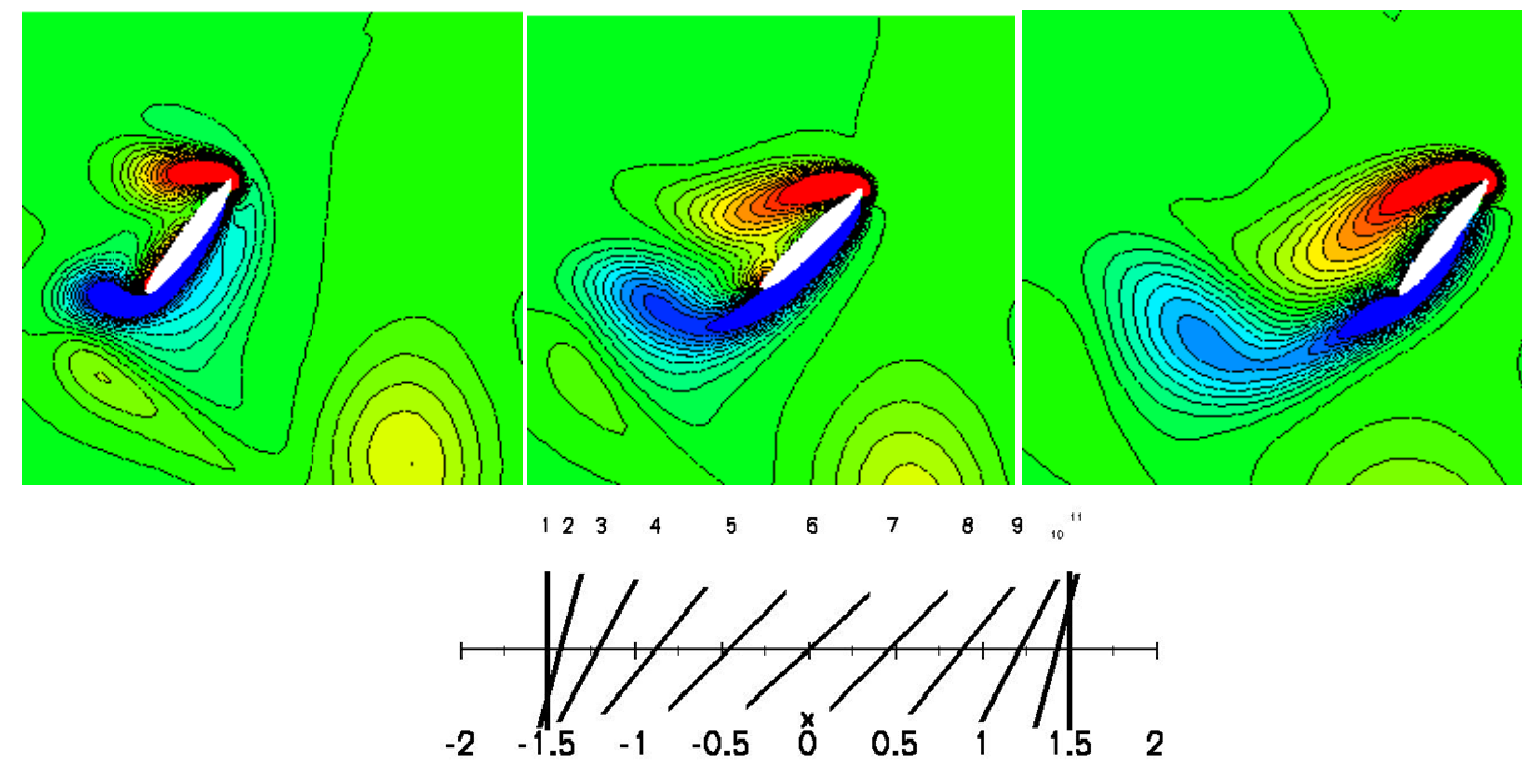

Figure 6. Delayed Stall: Illustration of unsteady delayed stall mechanism and the LEV with vorticity countours (red: counter-clockwise, blue: clockwise) These snapshots correspond to points 4, 6, and 8 respectively. 

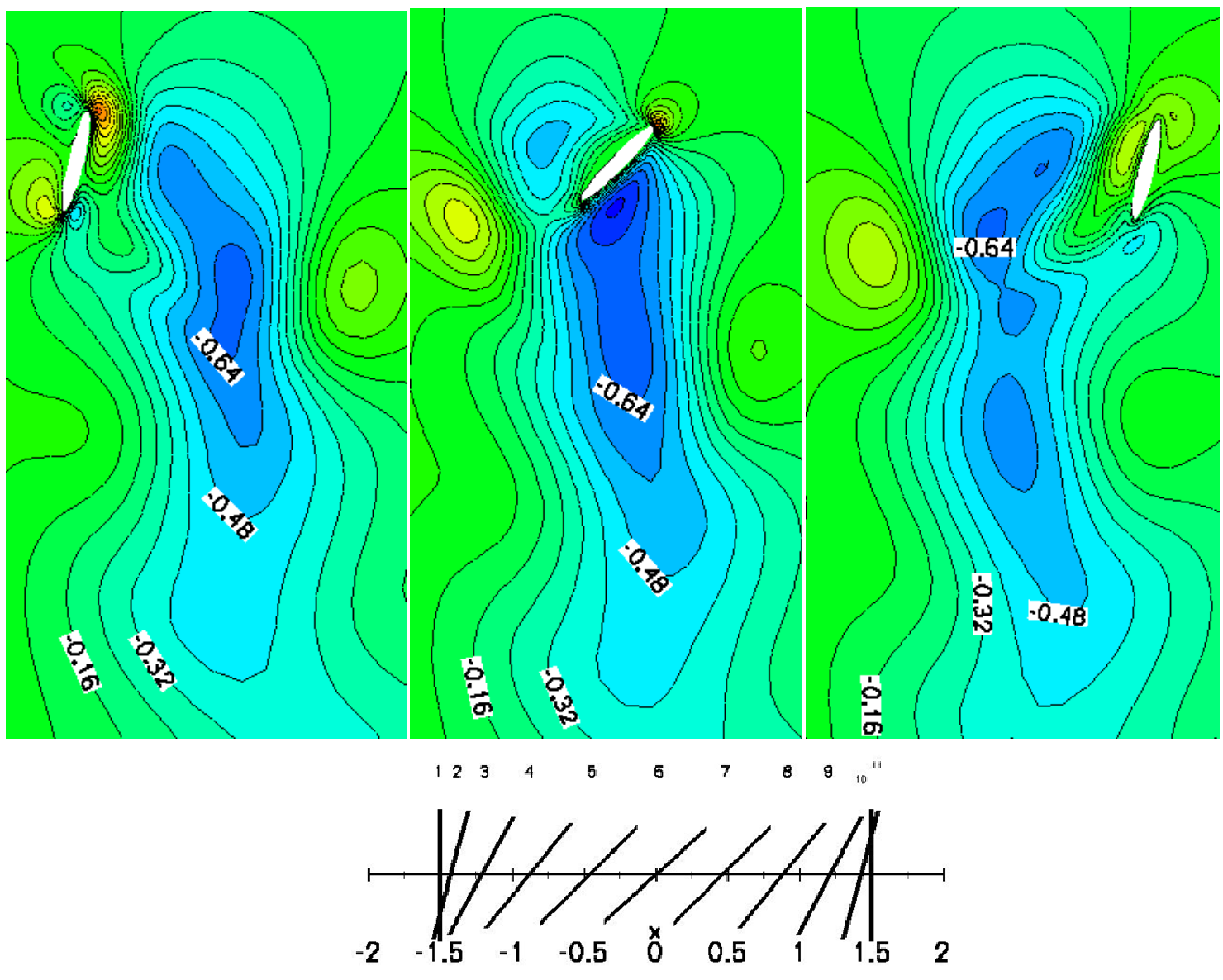

Figure 7. Illustration of the jet flow feature which develops in the path of the airfoil. Velocity contours are displayed (red: positive, blue: negative) with select percentages of the maximum translational velocity marked. These snapshots would correspond to points 2,6 , and 10 respectively.

The last unsteady flow mechanism is a jet-like flow feature present in the path of the airfoil where a persistent downward velocity develops. These regions within the jet are of comparable magnitude to the maximum translational velocity of the airfoil itself and are influential for a large segment of the cycle, in this case roughly from point 4 to point 9 in Figure 7. This jet is the cause of the asymmetry seen between forward and backstrokes, see Figure 3, and is due to the fact that the jet is slightly off centered in the direction in which the motion was started. The jet also explains the local minimum, near point 5 which will be referred to as the wake valley. If the angle of attack is low (higher angular amplitudes), or the jet strength is stronger (shorter plunging amplitudes mean the jet decays less between encounters) then this wake valley will become deeper. To support the argument of the jet's influence $C_{L}$ has been plotted during the first two cycles as well as the $15^{\text {th }}$ cycle where the differences between previous cycles has largely stopped at the spatial and temporal resolutions used (Figure 3). Note that because the simulation starts with a large discontinuity in airfoil velocity, and the plots are shifted such that the force histories start when the airfoil is at the end of its translation, the $1^{\text {st }}$ cycle does not imply no wake. Rather it serves to provide credibility to the claim the jet is at least partly responsible for the aerodynamic performance recorded (i.e. the LEV is not the only factor) as it is clear that as the jet changes strength, the aerodynamic characteristics respond in a noticeable fashion.

\section{Kinematics}

1. Plunging Amplitude $\left(2 h_{a} / c\right)$

Interestingly, the plunging amplitude, and with it the reduced frequency, had the smallest influence on lift and required power throughout the study. Since the peak translational velocity of the airfoil is equal for all cases, the fact 
that the plunging amplitude has little effect on the power required per cycle is to be expected. However, the effect on lift of the unsteady flow mechanisms is less easily predicted as is their cumulative impact. It is readily apparent from the surrogate response of the design space, as seen by the small gradients found in the direction of the arrows in Figure 8 , that the plunging amplitude's influence is limited.

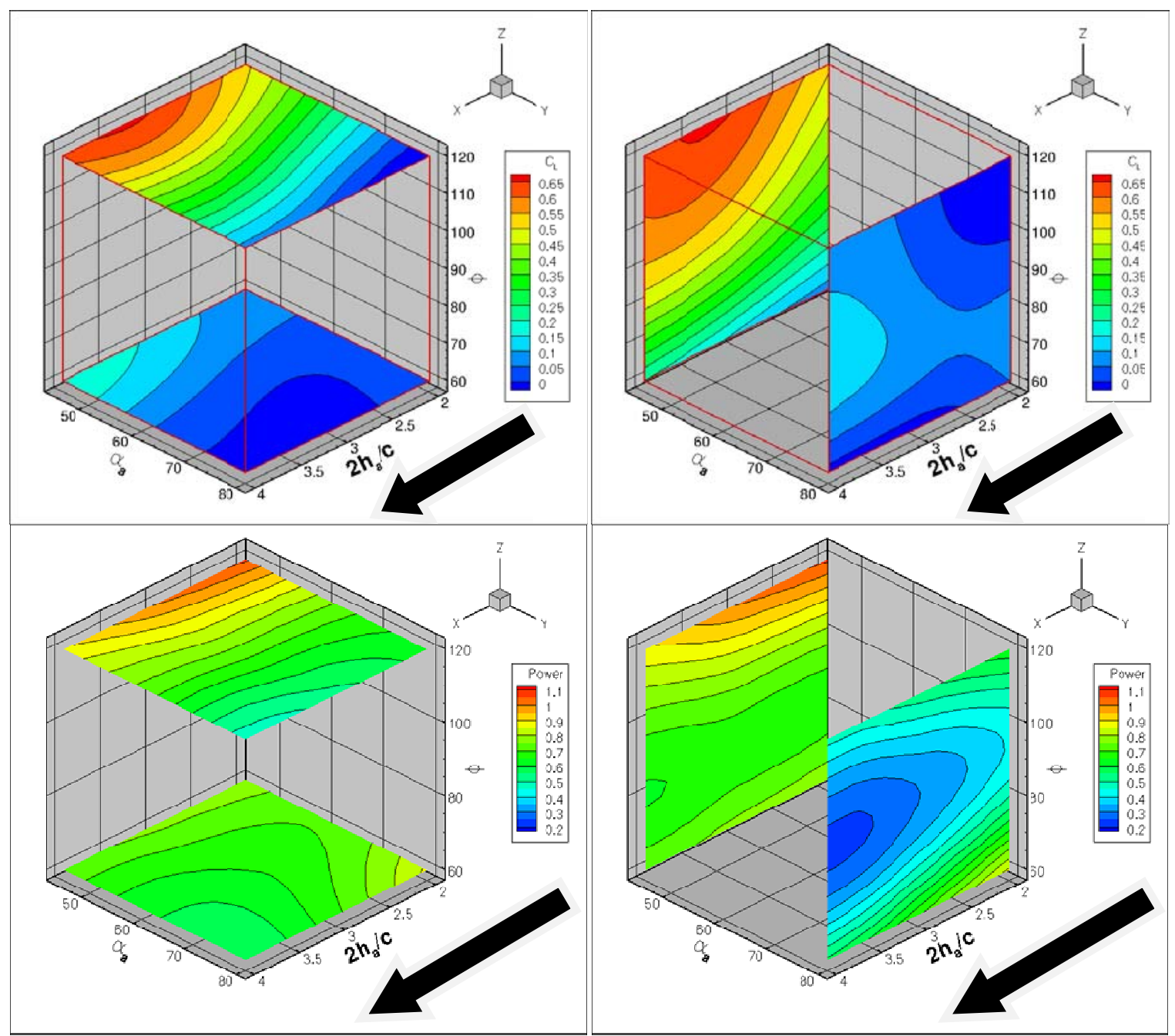

Figure 8. The lift coefficient (top) and power required (bottom) as approximated by the WAS illustrating the effect of the plunging amplitude. For the integrated effect over the entire cycle it is seen that this is the least sensitive of the design variables. Arrows show the direction of increasing plunging amplitude.

To gain a more comprehensive look on the plunging amplitude's influence, three force histories are shown in Figure 9. The first observation is that the wake capturing peak is slightly smaller as the plunging amplitude is increased. The most significant change though is in the wake valley. The wake valley is deepest when the plunging amplitude is shortest. As the plunging amplitude increases, the intensity of the jet found in the wake is able to decay before interaction with the airfoil. The influence of the jet is detrimental to the lift under these conditions, and a weaker jet corresponds to higher lift for airfoils at identical angles of attack and translational velocities. Moving on to the delayed stall peak, seen as the global maximums, there is little influence on the peak amplitude. The general implication then is to minimize the negative impact the persistent jet has after the wake capturing peak. In the context of plunging amplitude this means increasing the stroke length to encounter a weaker jet, or looking at it from another perspective this could be seen as approaching jets of similar intensity at higher angles of attack. 


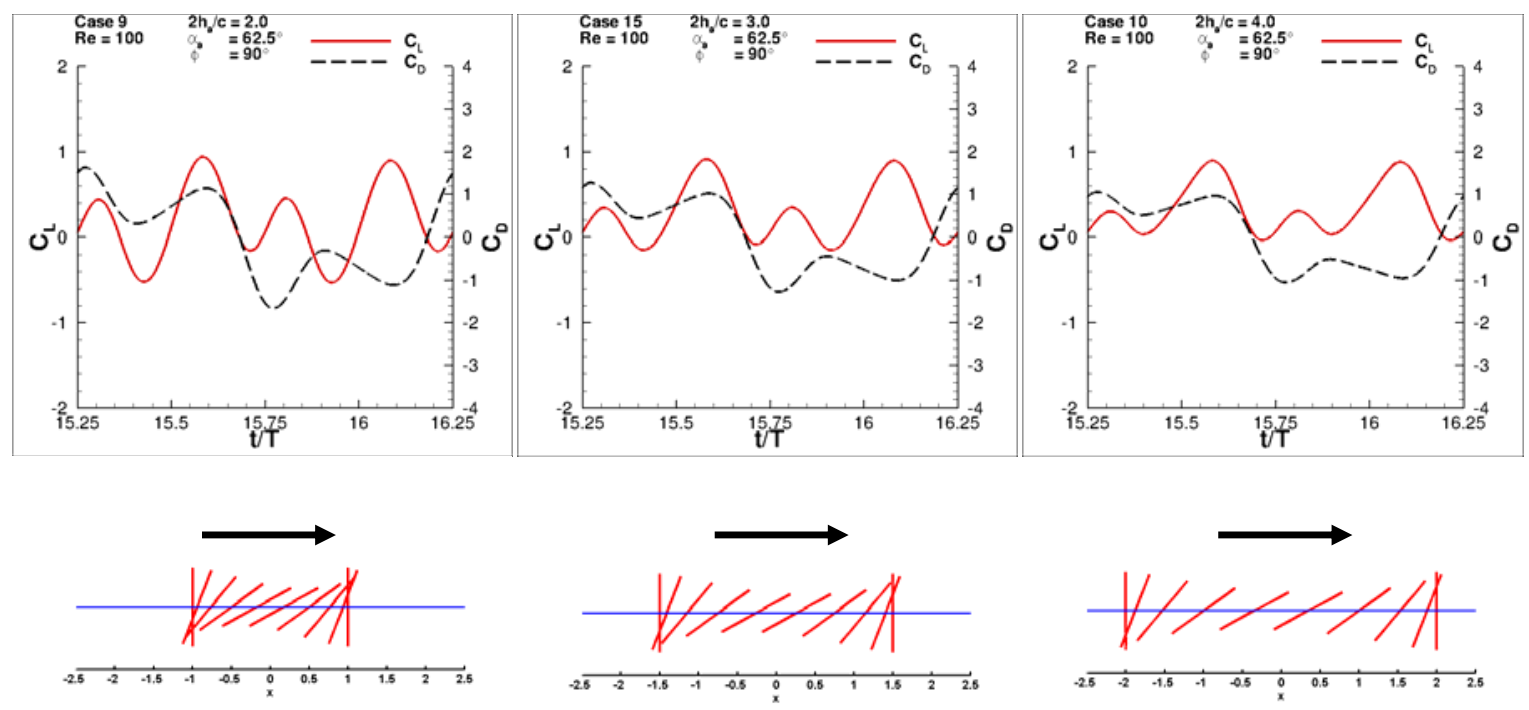

Figure 9. Snapshots of the effect of the plunging amplitude $(2.0,3.0,4.0)$ on the instantaneous force history.

\section{Angular Amplitude $\left(\alpha_{a}\right)$}

In direct contrast to the discussion concerning the plunging amplitude, the design space is quite sensitive to the angular amplitude, see Figure 10. In general it is found that lower angular amplitudes, thus higher angles of attack, lead to higher power requirements and lift. The power required result is expected as higher angles of attack will correspond to more drag, and consequently a larger power requirement. The lift result, while not unexpected, is the result of interactions of the unsteady aerodynamics and is not entirely intuitive. What is evident is the fact that higher lift and lower power required aspirations are in direct competition on either end of the angular amplitude spectrum.

Consider again the force histories, the focus this time is angular amplitude, see Figure 11. The first observation is that the amplitude of the wake capturing peak does not change much with angular amplitude. This is somewhat surprising considering that higher angular amplitudes lead to higher angular velocities which then interact with the wake behind the airfoil at the end of the translation. The root of why the angular amplitude matters so much is seen clearly in the wake valley and subsequent delayed stall peak. As the angular amplitude is increased, and the angles of attack decreased, the airfoil approaches the jet at less favorable orientations, making the wake valley deeper and wider. However, the delayed stall peak also decreases and so we have two effects acting in concert. The lower angles of attack experienced do provide diminished (and sometimes no) support for LEV formation and thus a much lower maximum lift value. At the same time, the higher angular amplitudes also provide a more streamlined body, producing less drag. It is seen that the rule of thumb is higher angles of attack, lower angular amplitudes, provide higher lift through a combination of jet interaction and LEV, but at the same time create a higher drag. 

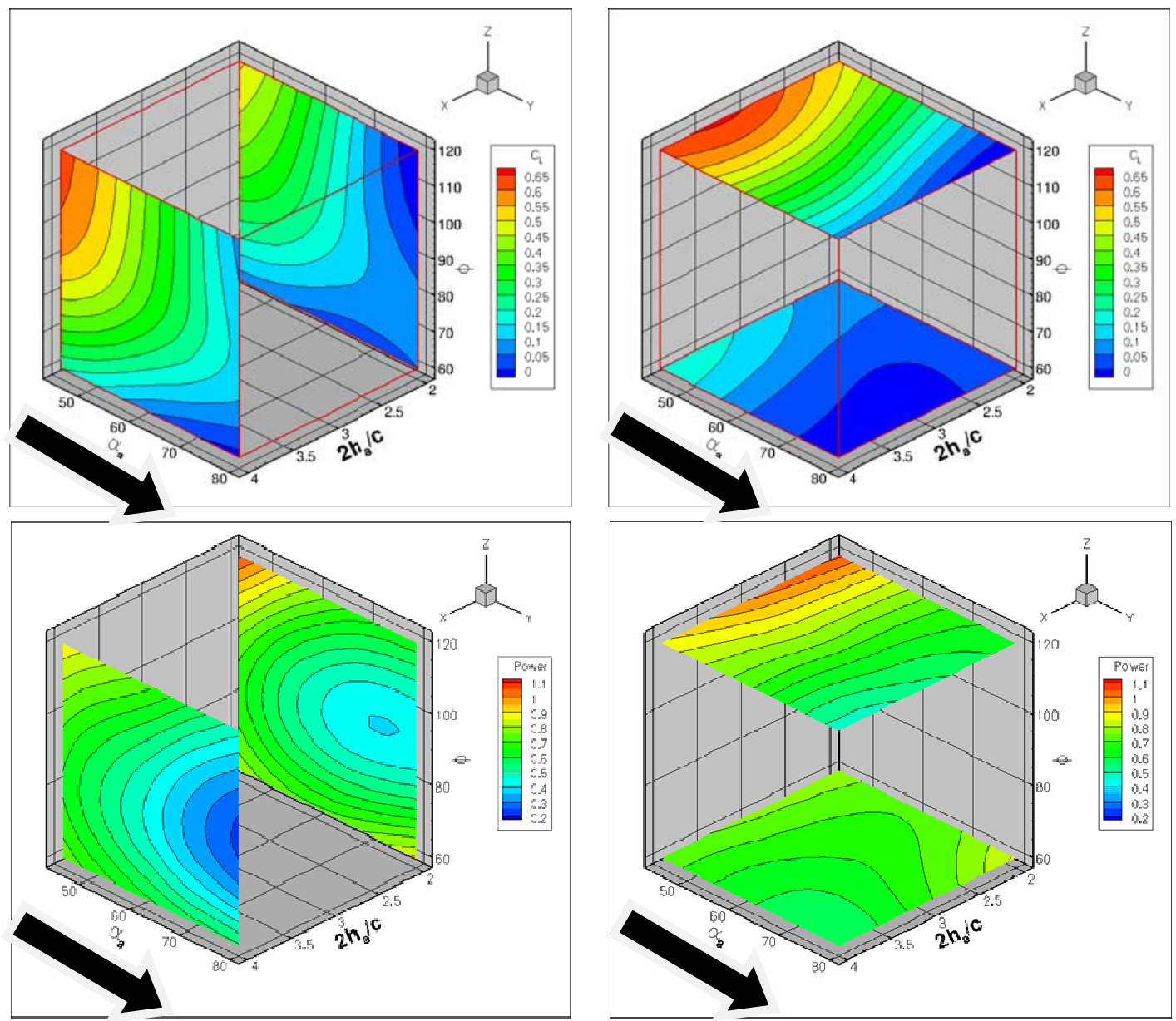

Figure 10. The lift coefficient (top) and power required (bottom) as approximated by the WAS illustrating the effect of the angular amplitude. A great deal of variance occurs with these changes and it is seen that in general lift coefficient over the whole cycle benefits from lower angular amplitudes. Arrows show the direction of increasing angular amplitude.

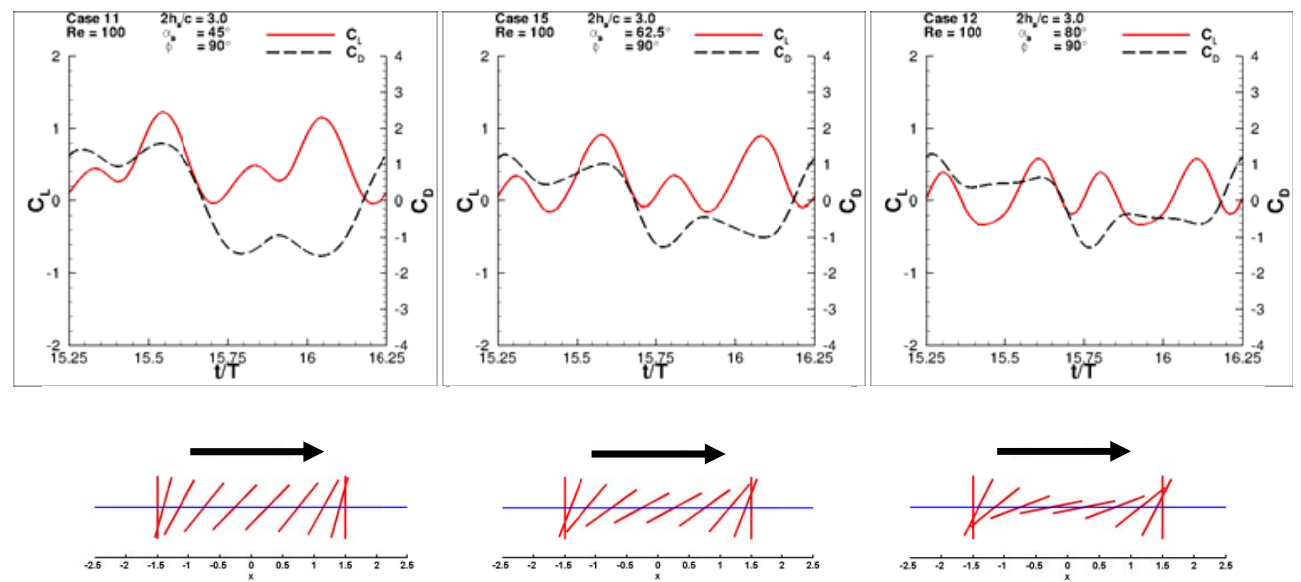

Figure 11. Snapshots of the effect of angular amplitude $(45,62.5,80)$ on the instantaneous force histories. 


\section{Phase Lag $(\Phi)$}

For the final design variable, phase lag, the general trend is for $\Phi>90^{\circ}$, or advanced rotation, to produce higher lift values. How this occurs will be explained shortly but is once again a combination of unsteady effects. It is also interesting to note that the phase lag's influence on the integrated lift is minimal when angular amplitudes are high. Yet, at higher angular amplitudes is exactly where largest influence is felt when considering the power required. The fact that the most efficient mode, from an energy input perspective, is when $\Phi \approx 90^{\circ}$. This puts the minimum angle of attack at the maximum translational velocity, and therefore less drag and power required.

Coupled with the force histories in Figure 13, a more complete picture emerges. During $\Phi>90^{\circ}$, or delayed rotation, The lift starts off negative and the wake capturing peak is shifted. From the illustrations of the airfoil positions, it can be seen that the airfoil is temporarily flying upside down and hence the negative lift immediately upon changing direction. When the wake capture peak does occur it is also being influenced by the jet, and at the higher angles of attack the interaction is beneficial. The delayed stall peak suffers. Though there is a higher angle of attack occurring at the maximum translational velocity, as compared to $\Phi=90^{\circ}$, the angular velocity is negative and not conducive to LEV formation.
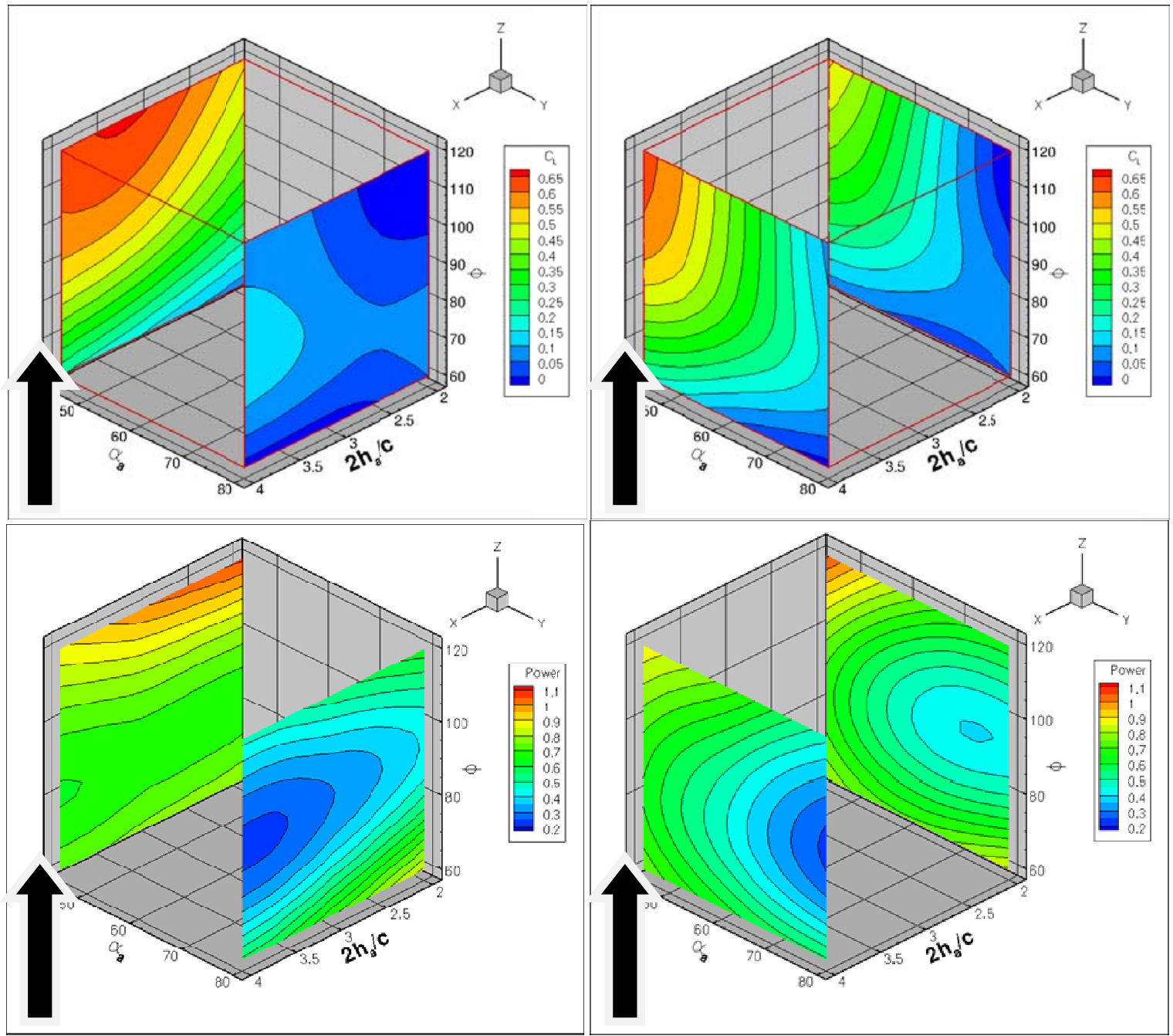

Figure 12. The lift coefficient (top) and power required (bottom) as approximated by the WAS illustrating the effect of the phase lag. Again a pronounced response in most areas of the design space. In general higher phase lags, corresponding to advanced rotation, are more beneficial from a lift point of view though that statement is not universally true, especially at higher angular amplitudes. Arrows show direction of increasing phase lag. 

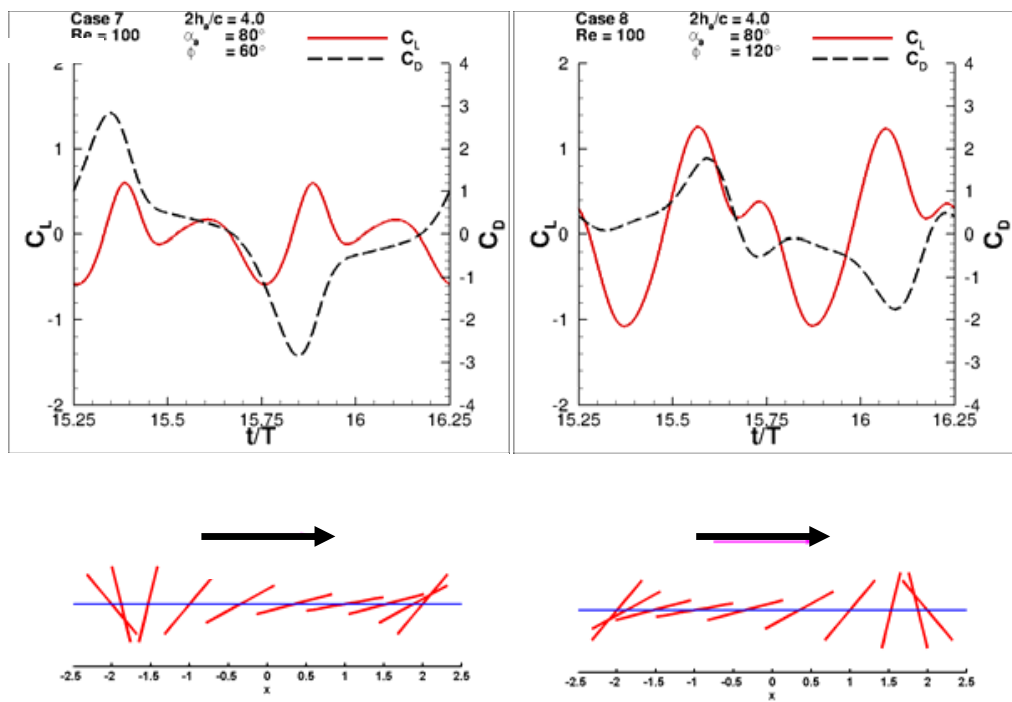

Figure 13. Force histories and airfoil positions illustrating delayed (left) and advanced (right) rotation. Here we see the utility in the advanced rotation as it keeps the lift levels high after the influence of the delayed stall has subsided.

For $\Phi>90^{\circ}$, or advanced rotation, the airfoil starts turning earlier such that a positive angle of attack is achieved upon wake capture producing favorable lift. This is immediately followed by a pronounced wake valley. It was seen that when $\Phi=90^{\circ}$, the wake valley could severely impede lift. That effect is exaggerated here where lower angles of attack encounter the jet. After that interaction, a very favorable delayed stall peak occurs as a higher angle of attack, with positive angular velocity, is present at the maximum translational velocity.

While one can see from the surrogates the consequences of changing the phase lag, the variable's influence is more than initially implied. As seen from the force histories, the influences on the wake capture, jet interaction, and delayed stall partially cancel out thus obscuring its overall importance.

\section{Global Sensitivity Analysis}

There is some sense of importance conveyed by the plots of the design space in Figure 8, Figure 10, and Figure 12. In low dimensions it may not be difficult to discern relative importance amongst the design variables. However, Figure 14 provides a more objective measure of influence. The global sensitivity indices are tabulated and a more quantifiable relationship can be determined. As expressed earlier, depending on whether instantaneous effects are important or whether the integrated result is sufficient, the role of phase lag could be underestimated. Regardless, within the design space examined, the plunging amplitude (and therefore the reduced frequency for normal hovering) plays a smaller role in determining the unsteady flow physics seen by an airfoil in normal hovering whereas the angular amplitude is a dominant factor.
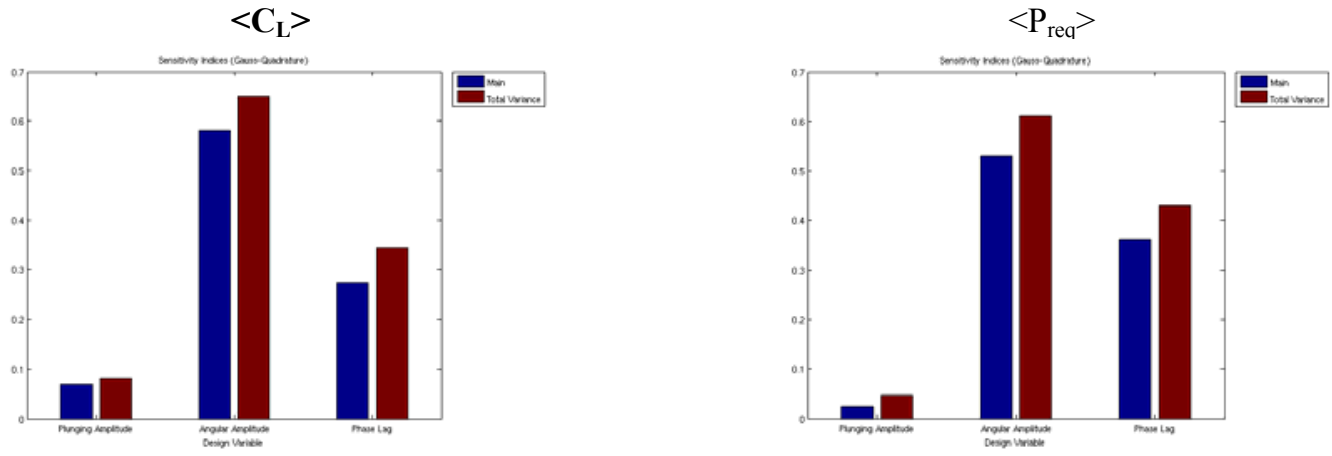

Figure 14. Sensitivity indices for $\left\langle\mathrm{C}_{\mathrm{L}}\right\rangle$ (left) and $\left\langle\mathrm{P}_{\text {req }}\right\rangle$ (right) in normal hovering. The main (blue) and total variances (red) from left to right: plunging amplitude, angular amplitude, phase lag. 


\section{Reynolds Number}

As the Reynolds number is increased, the vortex dynamics quickly lead to chaotic behavior. As seen in Figure 15, stronger vortices are created as the Reynolds number increases. These vortices persist and interact with each other over multiple stroke cycles which in turn leads to the chaotic force histories experienced by the airfoil, see Figure 16. Another implication of raising the Reynolds number is the strengthening of the jet-like flow feature which loses a stable configuration.

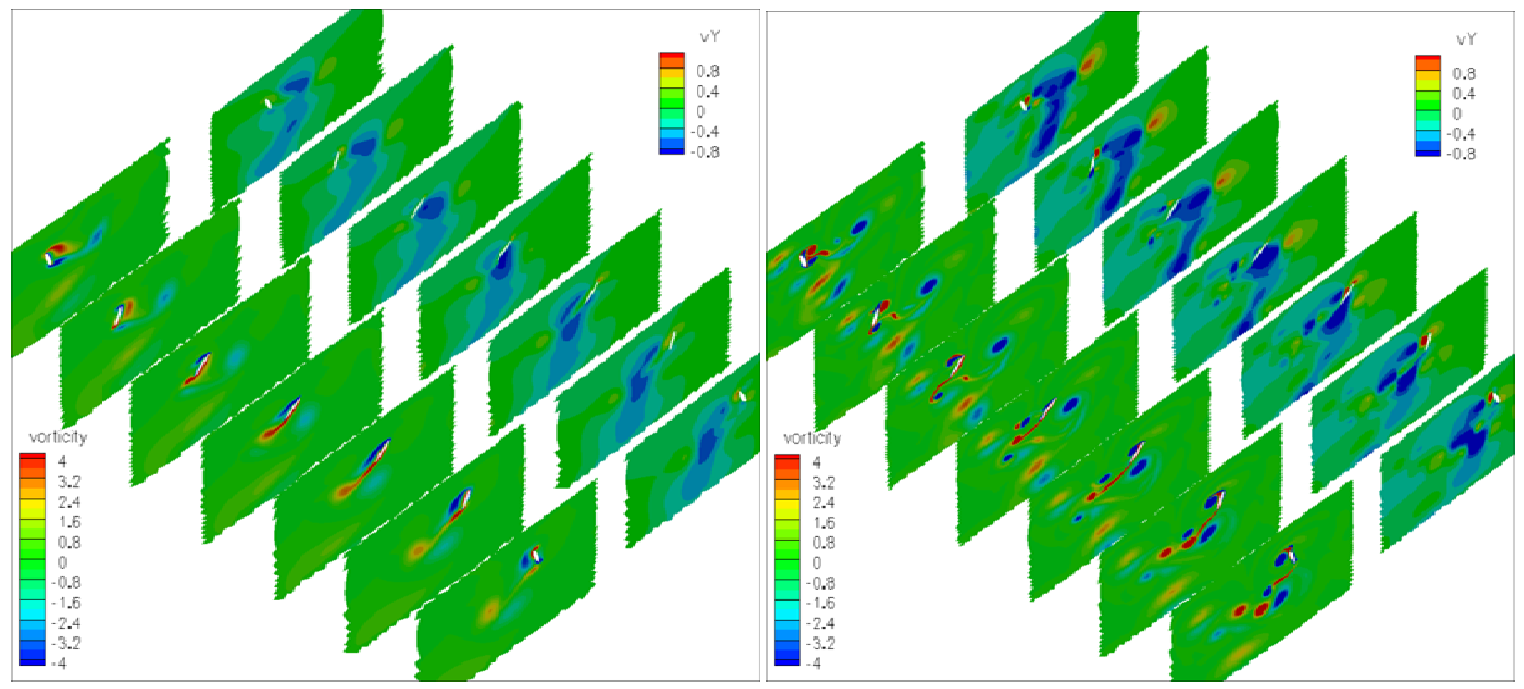

Figure 15. Vorticity and vertical velocity contour plots of normal hovering at $R e=100$ (left) and $R e=1000$ (right)

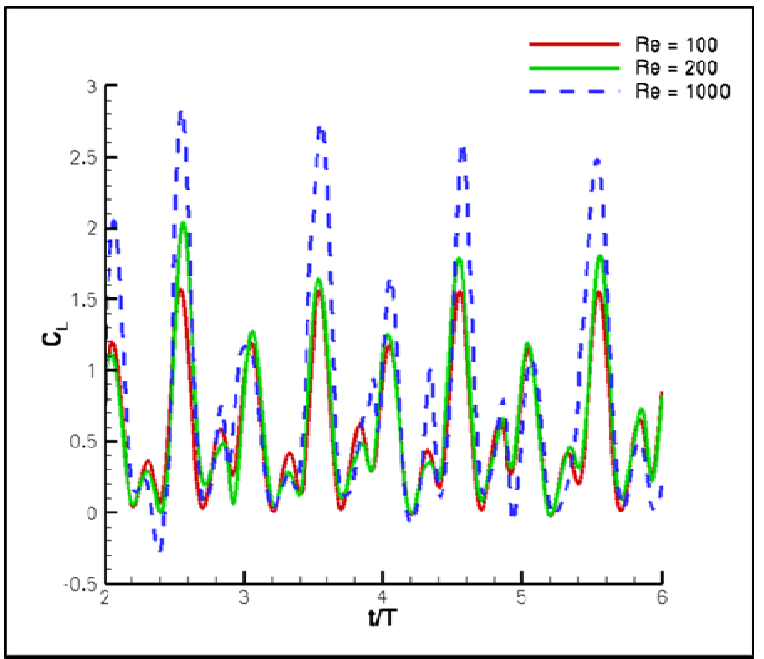

Figure 16. Reynolds number effect on $C_{L}$ going from $R e=100$ (Red), which displays a repeatable pattern, to $\mathrm{Re}=1000$ (Dashed-Blue) whose force histories vary substantially from stroke to stroke.

\section{Freestream Effect}

When a freestream is added to the flapping motions, Figure 17, the physics as well as the numerical issues associated with the calculations simplify significantly. The objective functions used for cases with freestream were again $\left\langle\mathrm{C}_{\mathrm{L}}>\right.$, though because all of the values were negative a high $\left\langle\mathrm{C}_{\mathrm{L}}\right\rangle$ is really just lower drag, and the viscous component, $\left\langle\mathrm{C}_{\mathrm{L}, \mathrm{vis}}>\right.$. The design spaces at $\mathrm{Re}=100$ and $\mathrm{Re}=1000$, where $\mathrm{Re}$ is based on flapping and the freestream, are shown in Figure 18 and Figure 19 respectively. The $\left\langle\mathrm{C}_{\mathrm{L}}\right\rangle$ values are shown on the same scale, and though the intensities do vary, the overall behavior is remarkably similar. Only subtle changes in the fluid physics take place as 
opposed to those encountered in the absence of a freestream. Also of note the only variable of significant importance, as reaffirmed by the sensitivity evaluations, is the angular amplitude. Once again the plunging amplitude does little to influence the overall $<\mathrm{C}_{\mathrm{L}}>$ picture. The phase lag drops in importance as well, because the freestream velocity is equivalent to the maximum translational velocity. Thus the angular amplitude dictates how much the flow is blocked, more drag at higher angular amplitudes, and where in the flapping cycle this occurs (the influence of the phase lag) matters very little.

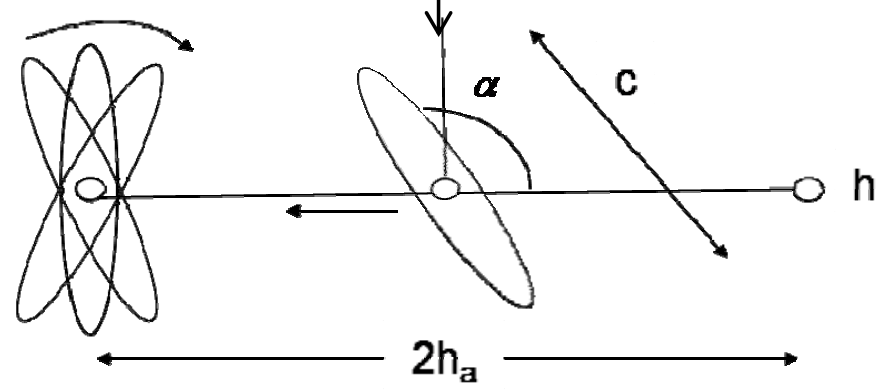

Figure 17. Hovering with with freestream $U_{\infty}$.

\section{$\operatorname{Re}=100$}
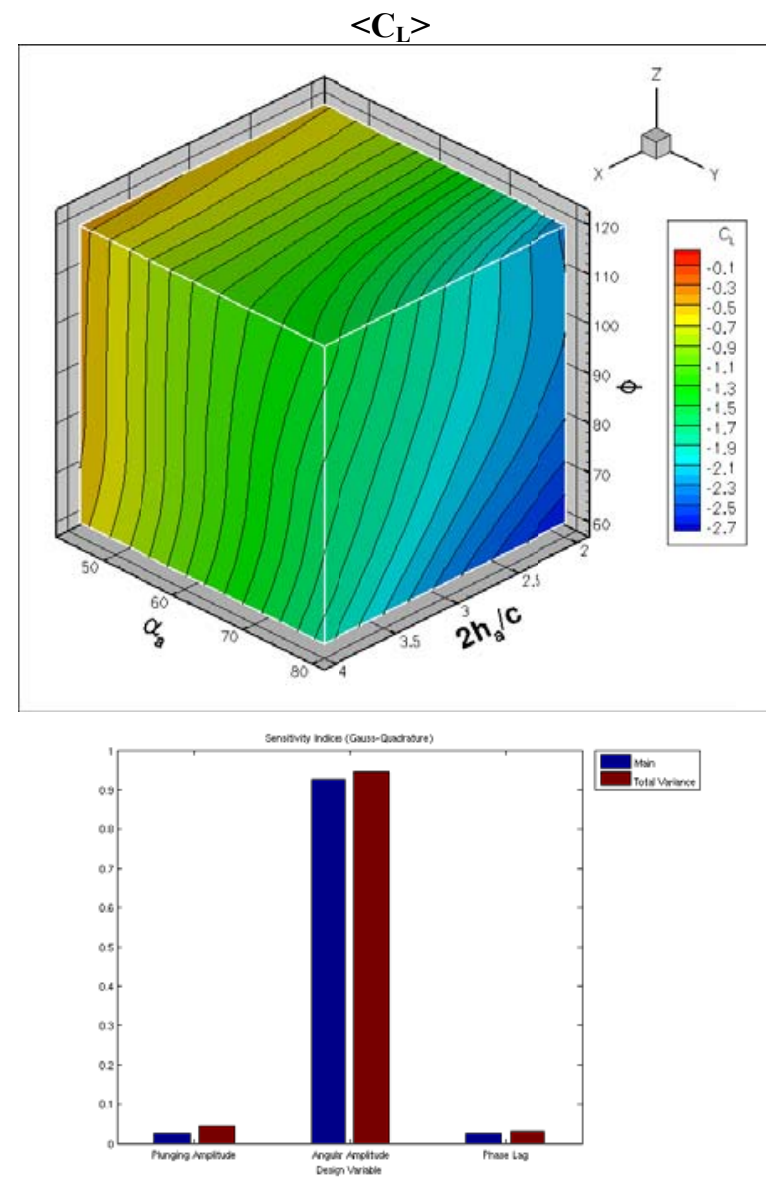
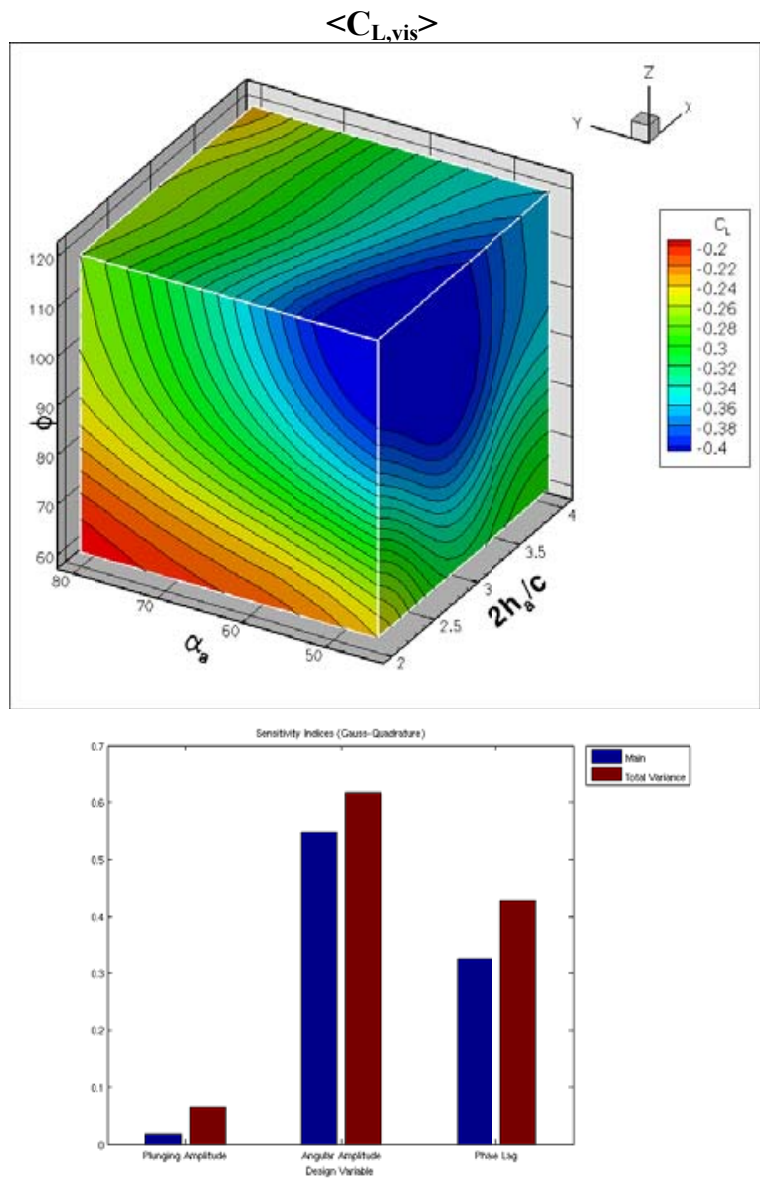

Figure 18. Design space/sensitivity analysis for freestream cases at $R e=100$. The total lift (left) and viscous component alone (right); note the axis are rotated 180 degrees from each other. The main (blue) and total (red) sensitivity indices for the plunging amplitude (left), angular amplitude (middle), and phase lag (right). 
Looking at the viscous component, note the scales have been adjusted between $\operatorname{Re}=100$ and $\operatorname{Re}=1000$ and the axis rotated relative to total lift as to enhance the regions of activity, once again the phase lag becomes relevant. The difference is at lower Reynolds numbers, the phase lag is important in and of itself, whereas the influence at higher Reynolds number is largely due to the coupling with the other design variables. The most visible discrepancy between Reynolds behaviors occurs when comparing the viscous component of lift at low phase angles and high angular amplitudes. At $\mathrm{Re}=100$ this region is red, Figure 18, corresponding to higher lift/lower drag. However at $\operatorname{Re}=1000$, this same region is light blue corresponding to lower lift/higher drag relative to the rest of the domain. This is due to the situation of having a portion of the cycle that is fairly sensitive to Reynolds numbers, where the airfoil is vertical, and then the stagnation flow which would have little influence on the force due to skin friction in the vertical direction. The overall impact does not influence the trends present in the total force experienced by the airfoil. Figure 21 gives a snapshot of the instantaneous force history vs Re as well as a plot of the integrated viscous force of the $15 \%$ thick ellipsoidal airfoil at 3 Reynolds numbers as compared to the theoretical flat plate Blasius solution.

\section{$\operatorname{Re}=1000$}
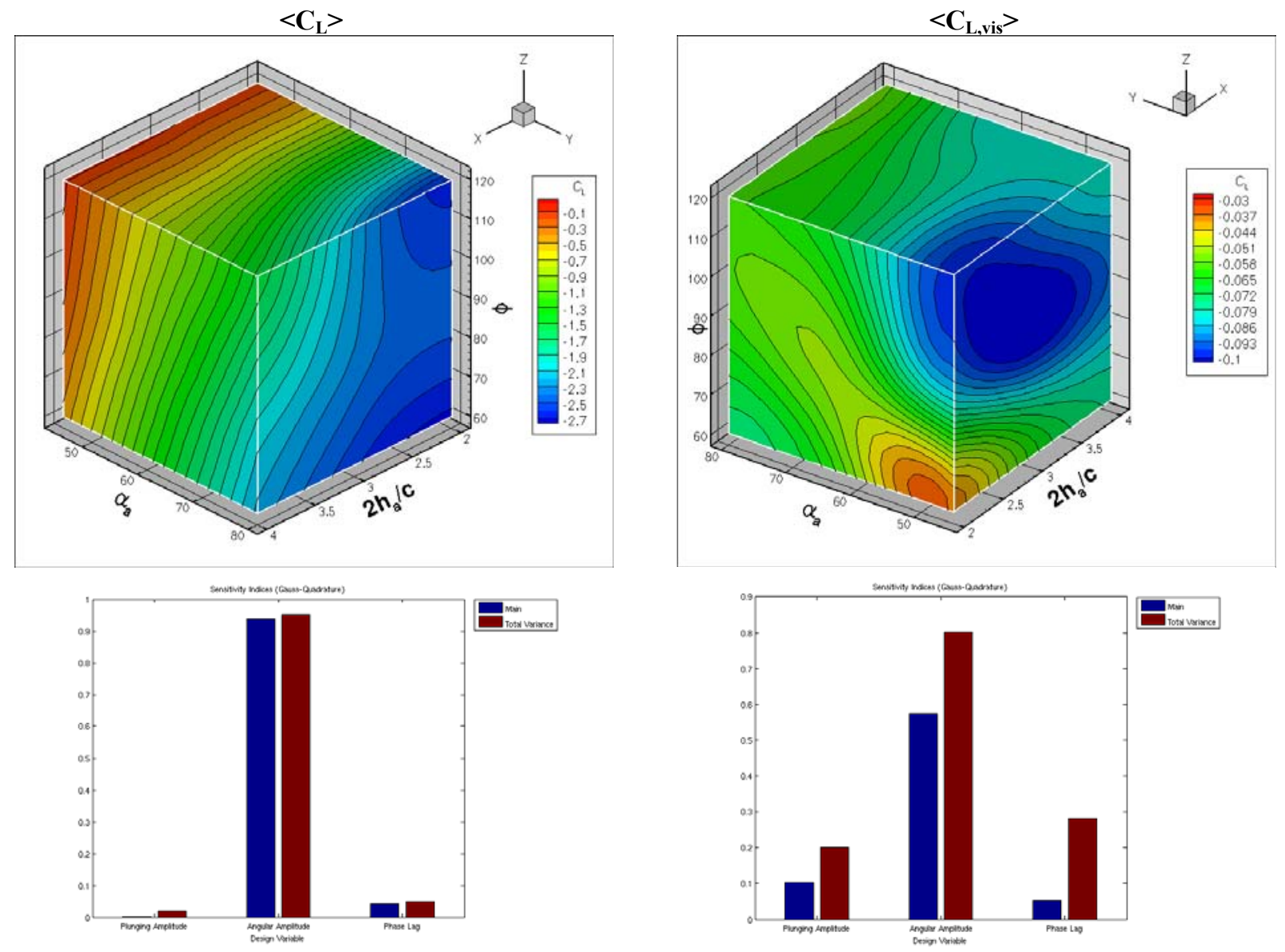

Figure 19. Design space/sensitivity analysis for freestream cases at $\mathbf{R e}=1000$. The total lift (left) and viscous component alone (right); note the axis are rotated 180 degrees from each other. The main (blue) and total (red) sensitivity indices for the plunging amplitude (left), angular amplitude (middle), and phase lag (right). 


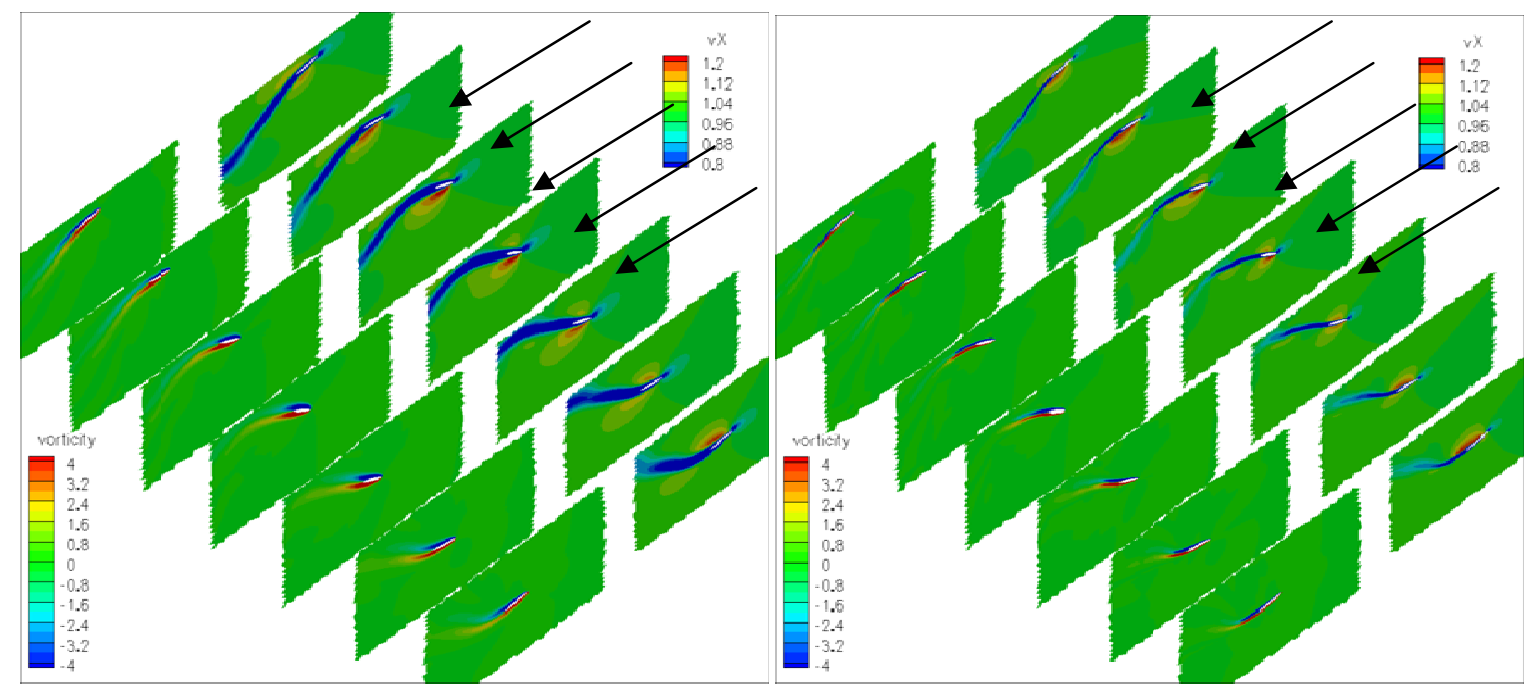

Figure 20. Flowfield shots of vorticity and velocity contours for $R e=100$ (left) and $R e=1000$ (right) with the freestream direction denoted by the arrows.
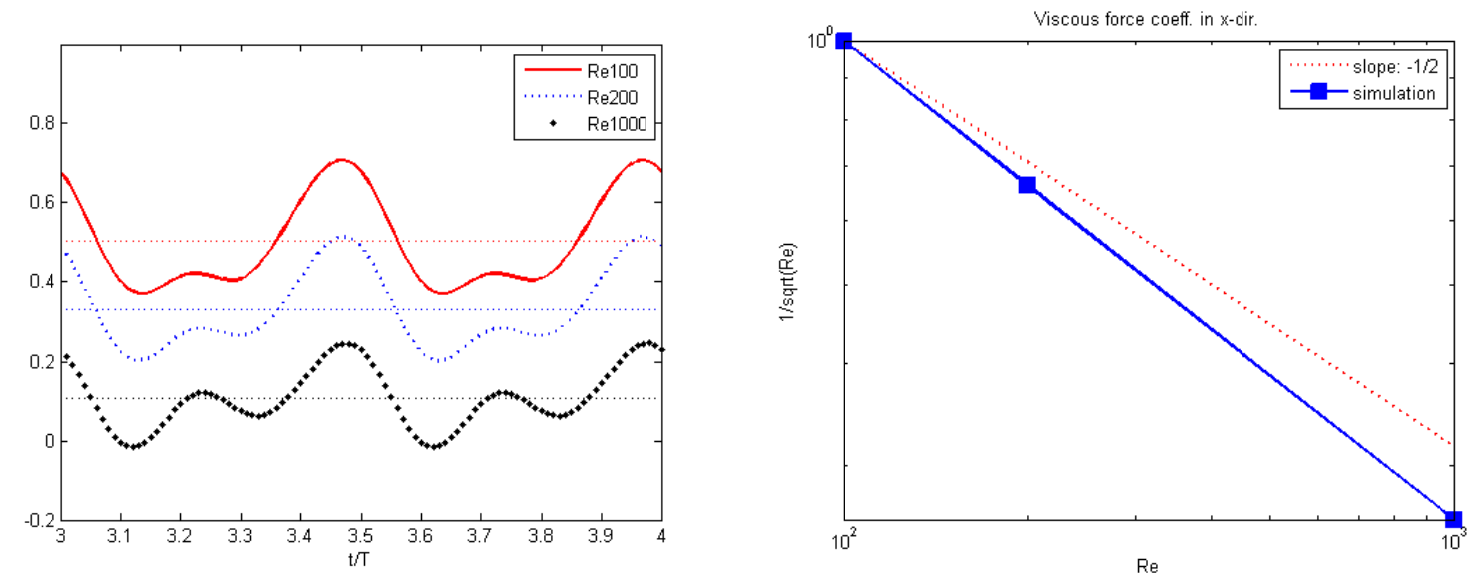

Figure 21. Time history Re effect on the $C_{L}$ with freestream present (left), and the $\left\langle C_{L, v i s}\right\rangle$ for the three Reynolds numbers: $\operatorname{Re}=100,200$, and 1000 in logarithmic axes (right). The dotted line represents $1 / \sqrt{ } \operatorname{Re}$ normalized by $R e=100$.

\section{Conclusions}

We conclude the investigation of the kinematics with freestream presence and Reynolds number effects. The normal hovering kinematics appeared to be largely uncoupled. In addition we see:

(i) The angular amplitude was by and large the most sensitive design variable.

(ii) If one was interested in the instantaneous effects of the variables, in addition to the integrated ones used to construct the surrogates, one would find the phase lag is under represented here because the tendency of its influences to partially cancel each other out.

(iii) Of interest is the fact that plunging amplitude, and hence the reduced frequency, does not play a vital role in the normal hovering kinematics within the bounds examined.

(iv) Even at Reynolds numbers of 1000, the force histories were non-repeatable, a consequence of the increasing frequency and strength of the vortex interactions with each other and the airfoil.

(v) The best integrated lift values were obtained from high phase lag values (advanced rotation) and low angular amplitudes (high angles of attack). However, the broad implications are that the wake capturing plays a small roll, whereas favorably influencing the delayed stall and jet interaction (a feature not 
emphasized in the literature to date) should be a motivating factor in kinematic design. Higher angles of attack being a primary concern for both.

From the power required standpoint, the least costly options appeared on the opposite side of the design space as the best lift scenarios, favoring the higher angular amplitudes (lower angles of attack) which allowed for a more streamlined flow.

None of the freestream cases examined were able to produce a positive lift. The flow physics and the numerical concerns were simplified by the presence of a preferred direction of motion.

(i) Once again the main variable of concern was the angular amplitude which dictated how much the freestream was blocked.

(ii) Both the plunging amplitude and phase lag became almost negligible factors in the overall forces felt.

(iii) When examining just the viscous component, both the plunging amplitude and phase lag become more significant as does the influence of these variables in combination.

(iv) As the Re was raised, the flow remained similar in structure in contrast to the normal hovering case which quickly tended towards chaos because of the increased number of vortices and their interactions.

The weighted average surrogates and associated global sensitivity evaluations provided an efficient method for mining the data for insight and trends not otherwise easily obtained. The sensitivity analysis provided a quick measure of the relative influence of the design variables while the behavior of the design space could be evaluated with the weighted average surrogate. The use of an ensemble of surrogates in the present context, while not likely to beat the best PRESS surrogate, mitigated the risk of having a surrogate model fit the training data well by chance but perform poorly at points not in the DOE.

Future work will look to include expanded kinematics, 3D flow physics, fluid structure interaction, and unsteady freestream effects.

Acknoledgements: The work supported here has been supported in part by the Air Force Office of Scientific Research's Multidisciplinary University Research Initiative (MURI) grant and by the Michigan/AFRL (Air Force Research Laboratory)/Boeing Collaborative Center in Aeronautical Sciences.

\section{Appendix}

\begin{tabular}{|c|c|c|c|c|c|c|c|c|c|}
\hline$\alpha_{0}=90^{\circ}$ & $2 h_{d} / c$ & $\alpha_{a}$ & $\Phi$ & $\begin{array}{c}\text { Normal } \\
R e=100 \\
<C_{L}>\end{array}$ & $\begin{array}{c}\text { Normal } \\
R e=100 \\
\left.<P_{\text {req }}\right\rangle\end{array}$ & $\begin{array}{c}\text { Free } \\
R e=100 \\
<C_{L, v i s}>\end{array}$ & $\begin{array}{c}\text { Free } \\
R e=100 \\
<C_{L}>\end{array}$ & $\begin{array}{c}\text { Free } \\
\operatorname{Re}=1000 \\
<C_{L, v i s}>\end{array}$ & $\begin{array}{c}\text { Free } \\
\operatorname{Re}=1000 \\
<C_{L}>\end{array}$ \\
\hline Case 1 & 2.0 & $45^{\circ}$ & $60^{\circ}$ & 0.07 & 0.79 & -0.24 & -0.74 & -0.038 & -0.38 \\
\hline Case 2 & 2.0 & $45^{\circ}$ & $120^{\circ}$ & 0.51 & 1.06 & -0.43 & -0.58 & -0.098 & -0.12 \\
\hline Case 3 & 2.0 & $80^{\circ}$ & $60^{\circ}$ & 0.05 & 0.86 & -0.19 & -2.70 & -0.073 & -2.73 \\
\hline Case 4 & 2.0 & $80^{\circ}$ & $120^{\circ}$ & -0.05 & 0.63 & -0.27 & -2.32 & -0.061 & -2.64 \\
\hline Case 5 & 4.0 & $45^{\circ}$ & $60^{\circ}$ & 0.20 & 0.74 & -0.29 & -0.56 & -0.076 & -0.43 \\
\hline Case 6 & 4.0 & $45^{\circ}$ & $120^{\circ}$ & 0.62 & 0.89 & -0.36 & -0.44 & -0.079 & -0.07 \\
\hline Case 7 & 4.0 & $80^{\circ}$ & $60^{\circ}$ & -0.02 & 0.56 & -0.27 & -1.87 & -0.076 & -2.23 \\
\hline Case 8 & 4.0 & $80^{\circ}$ & $120^{\circ}$ & 0.08 & 0.47 & -0.23 & -1.44 & -0.057 & -1.53 \\
\hline Case 9 & 2.0 & $62.5^{\circ}$ & $90^{\circ}$ & 0.18 & 0.44 & -0.30 & -1.17 & -0.056 & -0.83 \\
\hline Case 10 & 4.0 & $62.5^{\circ}$ & $90^{\circ}$ & 34 & 0.44 & -0.32 & -1.08 & -0.079 & -1.05 \\
\hline Case 11 & 3.0 & $45^{\circ}$ & $90^{\circ}$ & 50 & 0.70 & -0.40 & -0.50 & -0.105 & -0.11 \\
\hline Case 12 & 3.0 & $80^{\circ}$ & $90^{\circ}$ & 07 & 0.30 & -0.28 & -2.02 & -0.066 & -2.30 \\
\hline Case 13 & 3.0 & $62.5^{\circ}$ & $60^{\circ}$ & 0.02 & 0.65 & -0.27 & -1.37 & -0.079 & -1.42 \\
\hline Case 14 & 3.0 & $62.5^{\circ}$ & $120^{\circ}$ & 0.36 & 0.73 & -0.32 & -1.11 & -0.074 & -0.96 \\
\hline Case 15 & 3.0 & $62.5^{\circ}$ & $90^{\circ}$ & 0.28 & 0.44 & -0.32 & -1.13 & -0.064 & -0.98 \\
\hline Case 16 & 3.6 & $66^{\circ}$ & $78^{\circ}$ & 0.21 & 0.40 & -0.28 & -1.26 & -0.066 & -1.38 \\
\hline Case 17 & 2.9 & $47^{\circ}$ & $91^{\circ}$ & 0.48 & 0.67 & -0.40 & -0.56 & -0.106 & -0.18 \\
\hline Case 18 & 3.8 & $69^{\circ}$ & $115^{\circ}$ & 0.27 & 0.55 & -0.29 & -1.24 & -0.074 & -1.12 \\
\hline Case 19 & 2.0 & $52^{\circ}$ & $67^{\circ}$ & 0.11 & 0.66 & -0.24 & -0.88 & -0.038 & -0.51 \\
\hline Case 20 & 2.8 & $77^{\circ}$ & $98^{\circ}$ & 0.07 & 0.34 & -0.28 & -1.87 & -0.059 & -2.12 \\
\hline
\end{tabular}

Table A1. The design of experiment variable values for the 20 data points needed to construct the surrogate models as well as their time averaged $C_{L}$ and required power values for the normal hovering case and the $C_{L}$ values as well as the viscous contribution for freestream cases at $\operatorname{Re}=100$ and $\operatorname{Re}=1000$. 


\begin{tabular}{|l|l|l|l|l|l|l|l|l|l|}
\hline & KRG & PRS & RBNN & SVR1 & SVR2 & SVR3 & SVR4 & SVR5 & SVR6 \\
\hline$<\mathrm{C}_{\mathrm{L}}>$ & 0.075 & 0.103 & 0.182 & $\mathbf{0 . 0 4 8}$ & 0.104 & 0.128 & 0.139 & 0.087 & 0.120 \\
\hline$\left\langle\mathrm{P}_{\text {req }}>\right.$ & 0.107 & 0.131 & 0.361 & $\mathbf{0 . 0 6 6}$ & 0.190 & 0.291 & 0.300 & 0.276 & 0.313 \\
\hline
\end{tabular}

Table A2. Normal hovering PRESS calculations for the respective surrogate models giving a quantitative measure of the global fit. Lower is better. Kriging(KRG). Polynomial Response Surface (PRS). Radial Basis Neural Network (RBNN). Support Vector Regression (SVR). See Table 3 for model properties.

\section{References}

${ }^{1}$ Mueller, T.J., and DeLaurier, J.D., “Aerodynamics of Small Vehicles,” Annual Review of Fluid Mechanics, Vol. 35, 2003, pp. 89-111.

${ }^{2}$ Bohorquez, F., Samuel, P., Sirohi, J., Rudd, L., and Pines, D., "Design, Analysis and Hover Performance of a Rotary Wing Micro Air Vehicle," Journal of the American Helicopter Society, Vol. 48, No.2, 2003, pp. 80-90.

${ }^{3}$ Ho, S., Nassef, H., Pornsinsirirak, N., et al. "Unsteady Aerodynamics and Flow Control for Flapping Wing Flyers," Progress in Aerospace Sciences, Vol. 39, No. 8, 2003, pp. 635-681.

${ }^{4}$ Ellington, C.P., "Limitations on Animal Flight Performance," Journal of Experimental Biology, Vol. 160, 1991, pp. 71-91.

${ }^{5}$ Spedding, G.R., and Lissaman, P.B.S., "Technical Aspects of Microscale Flight Systems," Journal of Avian Biology, Vol. 29, No. 4, 1998, pp. 458-468.

${ }^{6}$ Shyy, W., Berg, M., and Ljungqvist, D., "Flapping and Flexible Wings for Biological and Micro Air Vehicles", Progress in Aerospace Sciences, Vol. 35, 1999, pp. 455-505.

${ }^{7}$ Ellington, C.P, "Novel Aerodynamics of Insect Flight: Applications to Micro-Air Vehicles," Jornal of Experimental Biology, Vol. 202, 1999, pp. 3439-3448.

${ }^{8}$ Norberg, U.M.L., "Structure, Form, and Function of Flight in Engineering and the Living World," Journal of Morphology, Vol. 252, No. 1, pp. 52-81.

${ }^{9}$ Wang, Z. J., "Dissecting Insect Flight," Annual Review of Fluid Mechanics, Vol. 37, 2005, pp. 183-210.

${ }^{10}$ Ansari, S.A., Zbikowski, R., and Knowles, K., “Aerodynamic Modeling of Insect-like Flapping Flight for Micro Air Vehicles," Progress in Aerospace Sciences, Vol. 42, 2006, pp. 129-172.

${ }^{11}$ Shyy, W., Lian, Y., Tang, J., Viieru, D., and Liu, H., Aerodynamics of Low Reynolds Number Flyers, Cambridge University Press, New York, 2008.

${ }^{12}$ Tang, J., Viieru, D., and Shyy, W., "Effects of Reynolds Number and Flapping Kinematics on Hovering Aerodynamics", AIAA paper 2007-129, Jan. 2007.

${ }^{13}$ Viieru, D., Tang, J., Lian, Y., Liu, H., and Shyy, W., "Flapping and Flexible Wing Aerodynamics of Low Reynolds Number Flight Vehicles", AIAA paper 2006-503, Jan. 2006.

${ }^{14}$ Viieru, D., Albertani, R., Shyy, W., and Ifju, P., "Effect of Tip Vortex on Wing Aerodynamics of Micro Air Vehicles", Journal of Aircraft, Vol. 42, 2005, p. 1530-1536.

${ }^{15}$ Shyy, W., and Liu, H., "Flapping Wings and Aerodynamic Lift: The Role of Leading-Edge Vortices," AIAA Journal, Vol. 45, No. 12 , 2007, pp. 2817-2819.

${ }^{16}$ Liu, H. and Kawachi, K., “A Numerical Study of Insect Flight”, Journal of Computational Physics, Vol. 146, 1998 , p. $124-156$.

${ }^{17}$ Wang, Z. J, "Computation of Insect Hovering", Mathematical Methods in the Applied Sciences, Vol. 24, 2001, p. 1515-1521.

${ }^{18}$ Wang, Z.J., Birch, J., and Dickinson M., "Unsteady Forces in Hovering Flight: Computation vs Experiments", Journal of Experimental Biology., Vol. 207, 2004, p. 449-460.

${ }^{19}$ Queipo, N., Haftka, R.T., Shyy, W., Goel, T. Vaidyanathan, R. and Tucker, P.K., "Surrogate-Based Analysis and Optimization," Progress in Aerospace Sciences, Vol. 41, 2005, pp. 1-25.

${ }^{20}$ Goel, T., Haftka, R.T., Shyy, W. and Watson, L.N., "Pitfalls of Using a Single Criterion for Selecting Experimental Designs," accepted for publication in International Journal for Numerical Methods in Engineering.

${ }^{21}$ Madsen, J.I., Shyy, W. and Haftka, R.T., "Response Surface Techniques for Diffuser Shape Optimization," AIAA Journal, Vol. 38, 2000, pp. 1512-1518.

${ }^{22}$ Goel, T., Dorney, D.J., Haftka, R.T., and Shyy, W., "Improving the Hydrodynamic Performance of Diffuser Vanes via Shape Optimization," accepted for publication in Computers and Fluids; also AIAA Paper No. 2007-5551, 43 ${ }^{\text {nd }}$ AIAA/ASME/SAE/ASEE Joint Propulsion Conference and Exhibit, 2007.

${ }^{23}$ Shyy, W., Papila, N., Vaidyanathan, R. and Tucker, P.K., "Global Design Optimization for Aerodynamics and Rocket Propulsion Components," Progress in Aerospace Sciences, Vol. 37, 2001, pp. 59-118.

${ }^{24}$ Luke, E.A., and George, T., "Loci: A Rule-Based Framework for Parallel Multi-Disciplinary Simulation Synthesis", Journal of Functional Programming, Vol. 15, No. 3, 2005, pp 477-502.

${ }^{25}$ Kamakoti, R., Thakur, S., Wright, J., and Shyy, W., "Validation of a new parallel all-speed CFD code in a rule-based framework for multidisciplinary applications", AIAA paper 2006-3063, June 2006. 
${ }^{26}$ Shyy, W., “ A Study of Finite Difference Approximations to Steady-State, Convection-Dominated Flow Problems”, Journal of Computational Physics, Vol. 57, No. 3, 1985, pp. 415-438.

${ }^{27}$ Shyy, W., Computational Modeling for Fluid Flow and Interfacial Transport, Elsevier, Amsterdam, 1994.

${ }^{28}$ Blazek, J., Computational Fluid Dynamics: Principles and Applications, Elsevier, Amsterdam, 2001.

${ }^{29}$ Thakur,S.T., and Wright, J., "Loci-STREAM: All-Speed CFD Solver for Arbitrary Polygonal Meshes in the Loci Framework", Streamline Numerics Inc., Gainesville, Florida, 2005(unpublished).

${ }^{30}$ Thomas, P.D., and Lombard, K., "The Geometric Conservation Law - A Link between Finite-Difference and Finite-Volume Methods of Flow Computation on Moving Grids", AIAA paper 1978-1208, July 1978.

${ }^{31}$ Kamakoti, R., and Shyy, W., "Evaluation of Geometric Conservation Law using Pressure-Based Fluid Solver and Moving Grid Technique", International Journal of Numerical Methods for Heat \& Fluid Flow, Vol. 14, No. 7, p. 851-865.

${ }^{32}$ Haftka, R.T., Scott, E.P., and Cruz, J.R., "Optimization and Experiments: a Survey," Applied Mechanics Review, Vol. 51, No. 7, 1998, pp. 435-448.

${ }^{33}$ Weis-Fogh, T., "Quick Estimates of Flight Fitness in Hovering Animals, Including Novel Mechanisms for Lift Production," Journal of Experimental Biology., Vol. 59, 1973, pp. 169-230.

${ }^{34}$ Weis-Fogh, T., "Energentics of Flight in Hummingbirds and in Drosophila", Journal of Experimental Biology, Vol. 56, p. 79104.

${ }^{35}$ Ellington, C.P., "The Aerodynamics of Hovering Insect Flight. III. Kinematics" Philosophical Transactions of the Royal Society of London, Series B, Vol. 305, p. 41-78.

${ }^{36}$ Viana, F.A.C., Haftka, R.T., Valder, S., Butkewitsch, S., and Leal, M.F., "Optimal Use of Multiple Surrogate For Reduced RMS Error in Meta-Model”, Proceedings of 2008 NSF Engineering Research Conference, Knoxville, USA, Jan. 7-10, 2008.

${ }^{37}$ Viana, F.A.C., Haftka, R.T., Valder, S., Butkewitsch, S, and Leal, M., "Ensemble of Surrogates: a Framework based on Minimization of the Mean Integrated Square Error," accepted for publication in AIAA Structures, Structures Dynamics, and Materials Conference, 2008.

${ }^{38}$ Myers, R.H., and Montgomery, D.C., Response Surface Methodology: Process and Product Optimization Using Designed Experiments, Wiley, New York, 2002.

${ }^{39}$ Cheng, B., and Titterington, D.M., "Neural Networks: a Review from a Statistical Perspective," Statistical Science, Vol. 9, 1994, pp. 2-54.

${ }^{40}$ Smola, A.J., and Scholkopf, B., “A Tutorial on Support Vector Regression,” Statistics and Computing, Vol. 14, 2004, pp.199222.

${ }^{41}$ Visbal, M.R., and Gaitonde, D.V., "High-Order-Accurate Methods for Complex Unsteady Subsonic Flows," AIAA Journal, Vol. 37, No. 10, 1999, pp. 1231-1239.

${ }^{42}$ Visbal, M.R., and Gaitonde, D.V., "On the Use of Higher-Order Finite-Difference schemes on Curvilinear and Deforming Meshes," Journal of Computational Physics, Vol. 181, No. 1, 2002, pp. 155-185. 